Jean-Michel Coron · Emmanuelle Crépeau

\title{
Exact boundary controllability of a nonlinear KdV equation with critical lengths
}

Received November 12, 2003

\begin{abstract}
We study the boundary controllability of a nonlinear Korteweg-de Vries equation with the Dirichlet boundary condition on an interval with a critical length for which it has been shown by Rosier that the linearized control system around the origin is not controllable. We prove that the nonlinear term gives the local controllability around the origin.
\end{abstract}

Keywords. Controllability, nonlinearity, Korteweg-de Vries

\section{Introduction}

Let us consider the following Korteweg-de Vries control system:

$$
(\mathrm{KdV})\left\{\begin{array}{l}
y_{t}+y_{x}+y_{x x x}+y y_{x}=0 \\
y(t, 0)=y(t, L)=0
\end{array}\right.
$$

For this control system, $L>0$ is given, the state is $y(\cdot):[0, L] \rightarrow \mathbb{R}$, and for the control one can take, for example, $u(\cdot)=y_{x}(\cdot, L) \in \mathbb{R}$. The Korteweg-de Vries equation serves to model various physical phenomena (see e.g. [19]), for example the propagation of small amplitude long water waves in a uniform channel. Let us recall that Bona and Winther have pointed out in [3] that the term $y_{x}$ in $(\mathrm{KdV})$ has to be added to model the water waves when $x$ denotes the spatial coordinate in a fixed frame. We are interested in the local controllability of $(\mathrm{KdV})$ around 0 . Rosier has proved in [12] that the control system $(\mathrm{KdV})$ is locally controllable around 0 provided that the length of the spatial domain is not critical.

Theorem 1 ([12, Theorem 1.3]). Let $T>0$, and assume that

$$
L \notin \mathcal{N}:=\left\{2 \pi \sqrt{\frac{j^{2}+l^{2}+j l}{3}} ; j, l \in \mathbb{N}^{*}\right\}
$$

J.-M. Coron: Université de Paris-Sud, Laboratoire ANEDP, Mathématique, UMR 8628, Bât. 425, 91405 Orsay Cedex, France; e-mail: Jean-Michel.Coron@math.u-psud.fr

E. Crépeau: INRIA, Projet SOSSO, Domaine de Voluceau-Rocquencourt, 78153 Le Chesnay Cedex; e-mail: Emmanuelle.Crepeau@inria.fr

Mathematics Subject Classification (2000): 93B05, 76B15 
Then there exists $r_{0}>0$ such that, for every $\left(y_{0}, y_{T}\right) \in L^{2}(0, L)^{2}$ with $\left\|y_{0}\right\|_{L^{2}(0, L)}<r_{0}$ and $\left\|y_{T}\right\|_{L^{2}(0, L)}<r_{0}$, there exists

$$
y \in C\left([0, T], L^{2}(0, L)\right) \cap L^{2}\left(0, T, H^{1}(0, L)\right)
$$

satisfying $(\mathrm{KdV})$ such that $y(0, \cdot)=y_{0}$ and $y(T, \cdot)=y_{T}$.

The aim of this paper is to study the local exact controllability around 0 of the nonlinear $\mathrm{KdV}$ equation when $L=2 k \pi \in \mathcal{N}$ (take $j=l=k$ in (1.1)). Our main theorem is the following one.

Theorem 2. Let $k$ be a positive integer and let $T>0$. There exists $r_{1}>0$ such that, for every $\left(y_{0}, y_{T}\right) \in L^{2}(0,2 k \pi)^{2}$ with $\left\|y_{0}\right\|_{L^{2}(0,2 k \pi)}<r_{1}$ and $\left\|y_{T}\right\|_{L^{2}(0,2 k \pi)}<r_{1}$, there exists

$$
y \in C\left([0, T], L^{2}(0,2 k \pi)\right) \cap L^{2}\left(0, T, H^{1}(0,2 k \pi)\right)
$$

satisfying $(\mathrm{KdV})$ with $L=2 k \pi$ such that $y(0, \cdot)=y_{0}$ and $y(T, \cdot)=y_{T}$.

When $L=2 k \pi$ the linearized control system of $(\mathrm{KdV})$ around 0 is

$$
(\mathrm{KdVL})\left\{\begin{array}{l}
y_{t}+y_{x}+y_{x x x}=0, \\
y(t, 0)=y(t, 2 k \pi)=0 .
\end{array}\right.
$$

It has been shown by Rosier in [12] that this linear control system is not controllable. To prove that the nonlinear term $y y_{x}$ gives the local controllability, a first approach could be to use the exact controllability of the nonlinear equation around nontrivial stationary solutions proved in [8] and to apply the method introduced in [6] (that is, the return method [4, 5] together with quasi-static deformations; see also [7] for this last point). But, with this method, it seems that one can only obtain the local exact controllability for large time. To prove Theorem 2 we use a different strategy that we briefly describe now. We first point out that in this theorem we may assume that $y_{0}=0$ : this follows easily from the invariance of (KdV) under the change of variables $\tau=T-t, \xi=2 k \pi-x$. Then we use the following result, due to Rosier, for the linearized control system (KdVL).

Theorem 3 ([12, Remark 3.6]). Let $T>0$ and

$$
H=\left\{y \in L^{2}(0,2 k \pi) ; \int_{0}^{2 k \pi}(1-\cos (x)) y d x=0\right\} .
$$

For $\left(y_{0}, y_{T}\right) \in H \times H$, there exists $y \in C\left([0, T], L^{2}(0,2 k \pi)\right) \cap L^{2}\left(0, T, H^{1}(0,2 k \pi)\right)$ satisfying $(\mathrm{KdVL})$ such that $y(0, \cdot)=y_{0}$ and $y(T, \cdot)=y_{T}$.

Then, as we shall prove in Section 2 the nonlinear term $y y_{x}$ allows us to "go" in the two directions $\pm(1-\cos (x))$ which are missed by the linearized control system (KdVL). Finally, in Section 3 we derive Theorem 2 from Section 2 by means of a fixed point theorem. 
Remark 4. For the other critical lengths, we believe that the same result holds. Note that the situation is more complicated in these other cases: there are now four noncontrollable directions for the linearized control system around 0 (see [12, proof of Lemma 3.5 and Remark 3.6]).

Remark 5. The method we use here (try to move in the directions which are missed by the linearized control system) is classical to study the local controllability of a control system in finite dimensions. Here we fix the time and perform a power series expansion, with the same scaling on the state and on the control. In finite dimensions, much more subtle tools have been introduced: for example different scalings on the components of the control and of the state as well as scaling on time. See e.g. [1, 2, 9, 10, 17, 18, 20] and the references therein.

Remark 6. One can find other results on the controllability of KdV control systems in [11, 13, 16] and the references therein.

\section{Motion in the $\pm(1-\cos (x))$ directions}

Let $L>0$. We first recall some properties proved by Rosier in [12] for the following linear KdV Cauchy problem:

$$
\begin{aligned}
& y_{t}+y_{x}+y_{x x x}=f, \\
& y(t, 0)=y(t, L)=0, \\
& y_{x}(t, L)=h(t), \\
& y\left(T_{0}, x\right)=y_{0}(x) .
\end{aligned}
$$

We adopt the notations of [12]. Let $A$ denote the operator $A w=-w^{\prime \prime \prime}-w^{\prime}$ defined on $\mathcal{D}(A):=\left\{w \in H^{3}(0, L) ; w(0)=w(L)=w_{x}(L)=0\right\}$ and let $\left(S(t)_{t \geq 0}\right)$ denote the semigroup of contractions associated with $A$ (see [12, Proposition 3.1]). For $T_{0}<T_{1}$, let

$$
B_{T_{0}, T_{1}}:=C\left(\left[T_{0}, T_{1}\right], L^{2}(0, L)\right) \cap L^{2}\left(T_{0}, T_{1}, H^{1}(0, L)\right)
$$

endowed with the norm

$$
\|y\|_{B_{T_{0}, T_{1}}}=\max \left\{\|y(t, \cdot)\|_{L^{2}(0, L)} ; t \in\left[T_{0}, T_{1}\right]\right\}+\left(\int_{T_{0}}^{T_{1}}\|y(t, \cdot)\|_{H^{1}(0, L)}^{2} d t\right)^{1 / 2} .
$$

Rosier has proved the following proposition.

Proposition 7 ([12, (Proofs of) Propositions 3.2 and 3.7]). Let $T_{0}<T_{1}$. There exist unique continuous linear maps $\Psi_{T_{0}, T_{1}}$ and $\delta_{T_{0}, T_{1}}$

$$
\begin{aligned}
\Psi_{T_{0}, T_{1}}: L^{2}(0, L) \times L^{2}\left(T_{0}, T_{1}\right) \times L^{1}\left(T_{0}, T_{1}, L^{2}(0, L)\right) & \rightarrow B_{T_{0}, T_{1}}, \\
\left(y_{0}, h, f\right) & \mapsto \Psi_{T_{0}, T_{1}}\left(y_{0}, h, f\right), \\
\delta_{T_{0}, T_{1}}: L^{2}(0, L) \times L^{2}\left(T_{0}, T_{1}\right) \times L^{1}\left(T_{0}, T_{1}, L^{2}(0, L)\right) & \rightarrow L^{2}\left(T_{0}, T_{1}\right), \\
\left(y_{0}, h, f\right) & \mapsto \delta_{T_{0}, T_{1}}\left(y_{0}, h, f\right),
\end{aligned}
$$


such that, for $y_{0} \in \mathcal{D}(A), h \in C^{2}\left(\left[T_{0}, T_{1}\right]\right)$ with $h\left(T_{0}\right)=0$ and $f \in C^{1}\left(\left[T_{0}, T_{1}\right], L^{2}(0, L)\right)$,

$$
\begin{gathered}
\Psi_{T_{0}, T_{1}}\left(y_{0}, h, f\right) \text { is the unique classical solution of 2.1) to 2.4, } \\
\delta_{T_{0}, T_{1}}\left(y_{0}, h, f\right)(t)=\left(\Psi_{T_{0}, T_{1}}\left(y_{0}, h, f\right)\right)_{x}(t, 0) .
\end{gathered}
$$

The function $\Psi_{T_{0}, T_{1}}\left(y_{0}, h, f\right)$ is called the mild solution of 2.1) to 2.4). For simplicity, we write $B$ for $B_{T_{0}, T_{1}}$ and $\Psi$ for $\Psi_{T_{0}, T_{1}}$ when $\left(T_{0}, T_{1}\right)=(0, T)$. Note that the existence of the continuous linear map $\delta_{T_{0}, T_{1}}$ shows that, with $y:=\Psi_{T_{0}, T_{1}}\left(y_{0}, h, f\right)$, " $y_{x}(t, 0)$ " makes sense in $L^{2}\left(T_{0}, T_{1}\right)$. For simplicity we shall write $y_{x}(t, 0)$ instead of $\delta_{T_{0}, T_{1}}\left(y_{0}, h, f\right)(t)$. Let $f \in L^{2}\left(T_{0}, T_{1}, L^{2}(0, L)\right)$. We say that $y:\left[T_{0}, T_{1}\right] \times[0, L] \rightarrow \mathbb{R}$ is a mild solution of

$$
y_{t}+y_{x}+y_{x x x}=f, \quad y(t, 0)=y(t, L)=0,
$$

if there exists $h \in L^{2}\left(T_{0}, T_{1}\right)$ such that $y$ is the mild solution of (2.1) to (2.4) with $y_{0}(x):=y\left(T_{0}, x\right)$. Note that it follows from the proof of Theorem 3 given in [12] that this theorem holds for mild solutions of (KdVL).

Until the end of this section we assume that

$$
L \in\left\{2 k \pi ; k \in \mathbb{N}^{*}\right\} .
$$

The aim of this section is to prove the following result.

Proposition 8. Let $T>0$. There exists $\left(u_{+}, v_{+}, w_{+}\right)$in $L^{2}(0, T)^{3}$ and $\left(u_{-}, v_{-}, w_{-}\right)$in $L^{2}(0, T)^{3}$ such that, if $\alpha_{ \pm}, \beta_{ \pm}, \gamma_{ \pm}$are the mild solutions of

$$
\begin{aligned}
& \alpha_{ \pm t}+\alpha_{ \pm x}+\alpha_{ \pm x x x}=0, \\
& \alpha_{ \pm}(t, 0)=\alpha_{ \pm}(t, L)=0, \\
& \alpha_{ \pm x}(t, L)=u_{ \pm}(t), \\
& \alpha_{ \pm}(0, x)=0
\end{aligned}
$$

of

$$
\begin{aligned}
& \beta_{ \pm t}+\beta_{ \pm x}+\beta_{ \pm x x x}=-\alpha_{ \pm} \alpha_{ \pm x}, \\
& \beta_{ \pm}(t, 0)=\beta_{ \pm}(t, L)=0 \\
& \beta_{ \pm x}(t, L)=v_{ \pm}(t) \\
& \beta_{ \pm}(0, x)=0
\end{aligned}
$$

and of

$$
\begin{aligned}
& \gamma_{ \pm t}+\gamma_{ \pm x}+\gamma_{ \pm x x x}=-\left(\alpha_{ \pm} \beta_{ \pm}\right)_{x}, \\
& \gamma_{ \pm}(t, 0)=\gamma_{ \pm}(t, L)=0 \\
& \gamma_{ \pm x}(t, L)=w_{ \pm}(t), \\
& \gamma_{ \pm}(0, x)=0
\end{aligned}
$$

then

$$
\alpha_{ \pm}(T, x)=0, \quad \beta_{ \pm}(T, x)=0, \quad \gamma_{ \pm}(T, x)= \pm(1-\cos (x)) .
$$


Remark 9. It would have been quite natural to look for the existence of $\left(u_{+}, v_{+}\right)$in $L^{2}(0, T)^{2}$ and of $\left(u_{-}, v_{-}\right)$in $L^{2}(0, T)^{2}$ such that, if $\alpha_{ \pm}, \beta_{ \pm}$are the mild solutions of 2.6 to 2.13, then

$$
\alpha_{ \pm}(T, x)=0, \quad \beta_{ \pm}(T, x)= \pm(1-\cos (x)) .
$$

The existence of such $\left(u_{ \pm}, v_{ \pm}\right)$would have also implied Theorem 2. Unfortunately, as proved in Corollary 19 below, such $\left(u_{ \pm}, v_{ \pm}\right)$do not exist. Roughly speaking, an expansion to the $2^{\text {nd }}$ order is not sufficient. We must go to the $3^{\text {rd }}$ order to get local controllability.

In order to prove Proposition 8 , let us first remark that $\left(u_{+}, v_{+}, w_{+}\right)$has the required properties if and only if $\left(u_{-}, v_{-}, w_{-}\right):=\left(-u_{+}, v_{+},-w_{+}\right)$does. Moreover, in order to prove the existence of $\left(u_{+}, v_{+}, w_{+}\right)$it suffices to prove the existence of $\left(u_{+}, v_{+}, \tilde{w}_{+}\right)$ in $L^{2}(0, T)^{3}$ such that, if $\alpha_{+}, \beta_{+}$and $\tilde{\gamma}_{+}$are the mild solutions of 2.6 to 2.17) with $\gamma_{+}:=\tilde{\gamma}_{+}$and $w_{+}:=\tilde{w}_{+}$, then

$\alpha_{+}(T, \cdot)=0, \quad \beta_{+}(T, \cdot)=0, \quad \int_{0}^{L} \tilde{\gamma}_{+}(T, x)(1-\cos (x)) d x=\|1-\cos (x)\|_{L^{2}(0, L)}^{2}$.

Indeed, by Theorem 3 (for mild solutions), there exists $w_{+}^{*}$ in $L^{2}(0, T)$ such that the mild solution $\gamma_{+}^{*}$ of

$$
\begin{aligned}
& \gamma_{+t}^{*}+\gamma_{+x}^{*}+\gamma_{+x x x}^{*}=0, \\
& \gamma_{+}^{*}(t, 0)=\gamma_{+}^{*}(t, L)=0, \\
& \gamma_{+x}^{*}(t, L)=w_{+}^{*}(t), \\
& \gamma_{+}^{*}(0, x)=0,
\end{aligned}
$$

satisfies

$$
\gamma_{+}^{*}(T, \cdot)=-\mathcal{P}_{H}\left(\tilde{\gamma}_{+}(T, \cdot)\right),
$$

where $\mathcal{P}_{H}$ denotes the orthogonal projection on $H$ for the $L^{2}$-scalar product. Then $u_{+}, v_{+}$ and $w_{+}:=\tilde{w}_{+}+w_{+}^{*}$ have the properties required by Proposition 8 (with $\gamma_{+}:=\tilde{\gamma}_{+}+\gamma_{+}^{*}$ ). Similarly, in order to prove the existence of $\left(u_{-}, v_{-}, w_{-}\right)$it suffices to prove the existence of $\left(u_{-}, v_{-}, \tilde{w}_{-}\right)$in $L^{2}(0, T)^{3}$ such that, if $\alpha_{-}, \beta_{-}$and $\tilde{\gamma}_{-}$are the mild solutions of (2.6) to 2.17 with $\gamma_{-}:=\tilde{\gamma}_{-}$and $w_{-}:=\tilde{w}_{-}$, then

$$
\alpha_{-}(T, \cdot)=0, \quad \beta_{-}(T, \cdot)=0, \quad \int_{0}^{L} \gamma_{-}(T, x)(1-\cos (x)) d x=-\|1-\cos (x)\|_{L^{2}(0, L)}^{2} .
$$

From 2.14, 2.15 and 2.17, one gets, using integration by parts (which can be easily justified by density arguments),

$$
\begin{aligned}
\int_{0}^{L} \gamma_{ \pm}(T, x)(1-\cos (x)) d x & =\int_{0}^{T} \int_{0}^{L} \gamma_{ \pm t}(t, x)(1-\cos (x)) d x d t \\
& =\int_{0}^{T} \int_{0}^{L}\left(-\gamma_{ \pm x}-\gamma_{ \pm x x x}-\left(\alpha_{ \pm} \beta_{ \pm}\right)_{x}\right)(1-\cos (x)) d x d t \\
& =\int_{0}^{T} \int_{0}^{L} \alpha_{ \pm} \beta_{ \pm} \sin (x) d x d t
\end{aligned}
$$

Hence Proposition 8 is a consequence of the following proposition. 
Proposition 10. Let $T>0$. There exists $(u, v)$ in $L^{2}(0, T)^{2}$ such that, if $\alpha, \beta$ are the mild solutions of

$$
\begin{aligned}
& \alpha_{t}+\alpha_{x}+\alpha_{x x x}=0, \\
& \alpha(t, 0)=\alpha(t, L)=0, \\
& \alpha_{x}(t, L)=u(t), \\
& \alpha(0, x)=0,
\end{aligned}
$$

and of

$$
\begin{aligned}
& \beta_{t}+\beta_{x}+\beta_{x x x}=-\alpha \alpha_{x}, \\
& \beta(t, 0)=\beta(t, L)=0, \\
& \beta_{x}(t, L)=v(t), \\
& \beta(0, x)=0,
\end{aligned}
$$

then

$$
\begin{gathered}
\alpha(T, \cdot)=0, \quad \beta(T, \cdot)=0, \\
\int_{0}^{T} \int_{0}^{L} \alpha \beta \sin (x) d x d t \neq 0 .
\end{gathered}
$$

Let $T>0$. Let $\alpha_{1} \in B$ be a mild solution of

$$
\begin{aligned}
& \alpha_{1 t}+\alpha_{1 x}+\alpha_{1 x x x}=0, \\
& \alpha_{1}(t, 0)=\alpha_{1}(t, L)=0,
\end{aligned}
$$

such that

$$
\int_{0}^{L} \alpha_{1}(0, x)(1-\cos (x)) d x=0 .
$$

Let us multiply $(2.29)$ by $1-\cos (x)$ and integrate the resulting equality on $[0, T] \times[0, L]$. Then, using integrations by parts together with 2.30 , and 2.31 , one gets

$$
\int_{0}^{L} \alpha_{1}(T, x)(1-\cos (x)) d x=0 .
$$

By Theorem 3 2.31) and 2.32], $\alpha_{1}$ can be extended to $[-T, 2 T] \times[0, L]$ in such a way that this extension is still a mild solution of (2.29)-(2.30) and satisfies

$$
\alpha_{1}(-T, x)=\alpha_{1}(2 T, x)=0 .
$$

Let $\beta_{1}:[0, T] \times[0, L] \rightarrow \mathbb{R}$ be a mild solution of

$$
\begin{aligned}
& \beta_{1 t}^{*}+\beta_{1 x}^{*}+\beta_{1 x x x}^{*}=-\alpha_{1} \alpha_{1 x}, \\
& \beta_{1}^{*}(t, 0)=\beta_{1}^{*}(t, L)=0 .
\end{aligned}
$$


By Theorem 3, there exists $\theta_{1} \in \mathbb{R}$ and a mild solution $\beta_{1} \in B_{-T, 2 T}$ of

$$
\begin{aligned}
& \beta_{1 t}+\beta_{1 x}+\beta_{1 x x x}=-\alpha_{1} \alpha_{1 x}, \\
& \beta_{1}(t, 0)=\beta_{1}(t, L)=0,
\end{aligned}
$$

such that

$$
\begin{aligned}
& \beta_{1}(t, x)=\beta_{1}^{*}(t, x)+\theta_{1}(1-\cos (x)), \quad \forall t \in[0, T], \\
& \beta_{1}(-T, x)=0, \quad \mathcal{P}_{H}\left(\beta_{1}(2 T, \cdot)\right)=0 .
\end{aligned}
$$

By Corollary 19 below, it follows that

$$
\beta_{1}(2 T, x)=0 .
$$

Let now $\alpha_{2}:[0, T] \times[0, L] \rightarrow \mathbb{R}$ be a mild solution of

$$
\begin{aligned}
& \alpha_{2 t}+\alpha_{2 x}+\alpha_{2 x x x}=0, \\
& \alpha_{2}(t, 0)=\alpha_{2}(t, L)=0,
\end{aligned}
$$

such that

$$
\alpha_{2}(0, x)=\alpha_{2}(T, x)=0 .
$$

By Theorem 3 , there exists a mild solution $\beta_{2}:[0, T] \times[0, L] \rightarrow \mathbb{R}$ of

$$
\begin{aligned}
& \beta_{2 t}+\beta_{2 x}+\beta_{2 x x x}=-\alpha_{2} \alpha_{2 x}, \\
& \beta_{2}(t, 0)=\beta_{2}(t, L)=0,
\end{aligned}
$$

such that

$$
\beta_{2}(0, x)=0, \quad \mathcal{P}_{H}\left(\beta_{2}(T, \cdot)\right)=0 .
$$

By Corollary 19 again,

$$
\beta_{2}(T, x)=0 .
$$

Similarly, by Theorem 3 and Corollary 19 , there exists a mild solution $\beta_{3}:[0, T] \times$ $[0, L] \rightarrow \mathbb{R}$ of

$$
\begin{aligned}
& \beta_{3 t}+\beta_{3 x}+\beta_{3 x x x}=-\left(\alpha_{1} \alpha_{2}\right)_{x} \\
& \beta_{3}(t, 0)=\beta_{3}(t, L)=0
\end{aligned}
$$

such that

$$
\beta_{3}(0, x)=0, \quad \beta_{3}(T, x)=0 .
$$

We extend $\alpha_{2}, \beta_{2}$ and $\beta_{3}$ to $[-T, 2 T] \times[0, L]$ by requiring

$$
\alpha_{2}(t, x)=\beta_{2}(t, x)=\beta_{3}(t, x)=0, \quad \forall t \in[-T, 0] \cup[T, 2 T] .
$$


Let us consider, for $\left(\rho_{1}, \rho_{2}\right) \in \mathbb{R}^{2}, \alpha:=\rho_{1} \alpha_{1}+\rho_{2} \alpha_{2}$ and $\beta:=\rho_{1}^{2} \beta_{1}+\rho_{2}^{2} \beta_{2}+\rho_{1} \rho_{2} \beta_{3}$. Let $u(t):=\alpha_{x}(t, L), v(t):=\beta_{x}(t, L)$ for $t \in[-T, 2 T]$. Then 2.19) to 2.21 and 2.23) to 2.25 hold in the mild sense and $\alpha(-T, x)=\beta(-T, x)=\alpha(2 T, x)=\beta(2 T, x)=0$. Now, assume that Proposition 10 is false. Then, for every $\left(\rho_{1}, \rho_{2}\right) \in \mathbb{R}^{2}$,

$$
\begin{aligned}
\int_{-T}^{2 T} & \int_{0}^{L} \alpha \beta \sin (x) d x d t \\
& =\int_{0}^{T} \int_{0}^{L}\left(\rho_{1} \alpha_{1}+\rho_{2} \alpha_{2}\right)\left(\rho_{1}^{2} \beta_{1}+\rho_{2}^{2} \beta_{2}+\rho_{1} \rho_{2} \beta_{3}\right) \sin (x) d x d t=0 .
\end{aligned}
$$

By looking at the coefficient of $\rho_{1}^{2} \rho_{2}$ in 2.45 , we get

$$
\int_{0}^{T} \int_{0}^{L}\left(\alpha_{1} \beta_{3}+\alpha_{2} \beta_{1}\right) \sin (x) d x d t=0 .
$$

For the time being the functions $\alpha_{1}, \beta_{1}, \beta_{2}$ and $\beta_{3}$ have been assumed to be real-valued. But, looking at the real and imaginary parts and applying the same trick we have used to get 2.46, we see that 2.46 also holds if these four functions take their values in $\mathbb{C}$. (Introduce arbitrary $\left(\rho_{1}, \rho_{2}\right)$. If $\alpha_{1}=a_{1}+i b_{1}$, consider $\tilde{\alpha}_{1}:=\rho_{1} a_{1}+\rho_{2} b_{1}$ etc.) Of course one says that $y=y^{1}+i y^{2}:\left[T_{0}, T_{1}\right] \times[0, L] \rightarrow \mathbb{C}$ is a mild solution of

$$
y_{t}+y_{x}+y_{x x x}=f, \quad y(t, 0)=y(t, L)=0,
$$

with $f=f^{1}+i f^{2} \in L^{1}\left(T_{0}, T_{1}, L^{2}(0, L, \mathbb{C})\right)$, if $y^{1}$ and $y^{2}$ are mild solutions of

$$
\begin{aligned}
y_{t}^{1}+y_{x}^{1}+y_{x x x}^{2}=f_{1}, & y^{1}(t, 0)=y^{1}(t, L)=0, \\
y_{t}^{2}+y_{2 x}^{2}+y_{x x x}^{2}=f^{2}, & y^{2}(t, 0)=y^{2}(t, L)=0 .
\end{aligned}
$$

Let $\lambda \in \mathbb{C}$. Let $y_{\lambda} \in C^{\infty}([0, L], \mathbb{C})$ be such that

$$
\begin{aligned}
& \lambda y_{\lambda}+y_{\lambda x}+y_{\lambda x x x}=0, \\
& y_{\lambda}(0)=y_{\lambda}(L)=0 .
\end{aligned}
$$

We take, for $t \in[0, T]$ and $x \in[0, L]$,

$$
\alpha_{1}(t, x)=e^{\lambda t} y_{\lambda}(x) .
$$

From 2.47), 2.48) and 2.49, we get 2.29) and 2.30). Multiplying (2.47) by $1-\cos (x)$, integrating the resulting equality on $[0, L]$ and using integrations by parts together with 2.48, we get

$$
\lambda \int_{0}^{L}(1-\cos (x)) y_{\lambda} d x=0
$$

By 2.49) and 2.50), if

$$
\lambda \neq 0,
$$


which will be assumed until the end of this section, 2.31 holds. Let $\tilde{A}$ denote the operator $A w=-w^{\prime \prime \prime}-w^{\prime}$ defined on $\mathcal{D}(\tilde{A}):=\left\{w \in H^{3}(0, L) ; w(0)=w(L)=0, w_{x}(0)=\right.$ $\left.w_{x}(L)\right\}$. Then $i \tilde{A}$ is a self-adjoint operator on $L^{2}(0, L)$ with compact resolvent. Hence, the spectrum $\sigma(\tilde{A})$ of $\tilde{A}$ is a discrete subset of $i \mathbb{R}$. Assume that

$$
\lambda \notin \sigma(\tilde{A}) .
$$

Then there exists one (and only one) $\phi_{\lambda} \in C^{\infty}([0, L], \mathbb{C})$ such that

$$
\begin{aligned}
& \lambda \phi_{\lambda}+\phi_{\lambda x}+\phi_{\lambda x x x}=y_{\lambda} \sin (x), \\
& \phi_{\lambda}(0)=\phi_{\lambda}(L)=0, \quad \phi_{\lambda x}(0)=\phi_{\lambda x}(L) .
\end{aligned}
$$

We multiply (2.53) by $y_{\lambda}(L-x)$, integrate on $[0, L]$, and use integrations by parts together with (2.5), 2.47), (2.48) and (2.54) to get

$$
\phi_{\lambda x}(L)\left(y_{\lambda x}(L)-y_{\lambda x}(0)\right)=0 .
$$

From now on we assume that

$$
y_{\lambda} \neq 0 .
$$

By 2.47, 2.48, 2.52, and 2.56,

$$
y_{\lambda x}(L) \neq y_{\lambda x}(0),
$$

which, together with 2.55], gives

$$
\phi_{\lambda x}(L)=0 .
$$

From 2.42, 2.43, 2.49, 2.53, 2.54) and 2.58, we get

$$
\frac{d}{d t} \int_{0}^{L} e^{\lambda t} \phi_{\lambda} \beta_{3} d x=\int_{0}^{L} \alpha_{1} \beta_{3} \sin (x) d x+\int_{0}^{L} \alpha_{1} \alpha_{2} \phi_{\lambda x} e^{\lambda t} d x .
$$

We also assume that

$$
2 \lambda \notin \sigma(\tilde{A}) .
$$

Then there also exists a unique $z_{\lambda} \in C^{\infty}([0, L], \mathbb{C})$ such that

$$
\begin{aligned}
& 2 \lambda z_{\lambda}+z_{\lambda x}+z_{\lambda x x x}=-y_{\lambda} y_{\lambda x}, \\
& z_{\lambda}(0)=z_{\lambda}(L)=0, \quad z_{\lambda x}(0)=z_{\lambda x}(L) .
\end{aligned}
$$

Define $\beta_{1}^{*}$ by

$$
\beta_{1}^{*}(t, x)=e^{2 \lambda t} z_{\lambda}(x)
$$


From 2.49) and 2.49) to 2.63), 2.33 and 2.34) hold. Let also (use 2.60) again) $\psi_{\lambda} \in$ $C^{\infty}([0, L], \mathbb{C})$ be the unique solution of

$$
\begin{aligned}
& 2 \lambda \psi_{\lambda}+\psi_{\lambda x}+\psi_{\lambda x x x}=z_{\lambda} \sin (x), \\
& \psi_{\lambda}(0)=\psi_{\lambda}(L)=0, \psi_{\lambda x}(0)=\psi_{\lambda x}(L) .
\end{aligned}
$$

Let

$$
\mu(x)=-\frac{1}{2} x \sin (x)+\frac{1}{12}(1-\cos (2 x))
$$

Then

$$
\begin{aligned}
& \mu_{x}+\mu_{x x x}=(1-\cos (x)) \sin (x), \\
& \mu(0)=\mu(L)=\mu_{x}(0)=0, \\
& \mu_{x}(L)=-L / 2 .
\end{aligned}
$$

From 2.37, 2.39, 2.40, 2.53, 2.54 and 2.63, to 2.68, we get

$$
\begin{aligned}
\frac{d}{d t} \int_{0}^{L}\left(e^{2 \lambda t} \psi_{\lambda}+\theta_{1} \mu\right) \alpha_{2} d x= & e^{2 \lambda t} \psi_{\lambda x}(L)\left(\alpha_{2 x}(t, L)-\alpha_{2 x}(t, 0)\right) \\
& -\frac{L}{2} \theta_{1} \alpha_{2 x}(t, L)+\int_{0}^{L} \alpha_{2} \beta_{1} \sin (x) d x .
\end{aligned}
$$

By (2.41) and (2.44),

$$
\int_{0}^{T}\left(\frac{d}{d t} \int_{0}^{L}\left(\left(e^{2 \lambda t} \psi_{\lambda}+\theta_{1} \mu\right) \alpha_{2}+e^{\lambda t} \phi_{\lambda} \beta_{3}\right) d x\right) d t=0 .
$$

From 2.59, 2.69) and 2.70, we get

$$
\begin{aligned}
\int_{0}^{T} \int_{0}^{L}\left(\alpha_{1} \beta_{3}+\alpha_{2} \beta_{1}\right) \sin (x) d x d t=-\int_{0}^{T} \int_{0}^{L} e^{\lambda t} \alpha_{1} \alpha_{2} \phi_{\lambda x} d x d t \\
\quad-\int_{0}^{T}\left(e^{2 \lambda t} \psi_{\lambda x}(L)\left(\alpha_{2 x}(t, L)-\alpha_{2 x}(t, 0)\right)-\frac{L}{2} \theta_{1} \alpha_{2 x}(t, L)\right) d t .
\end{aligned}
$$

Let (see 2.60) $\delta_{\lambda} \in C^{\infty}([0, L], \mathbb{C})$ be the unique solution of

$$
\begin{aligned}
& 2 \lambda \delta_{\lambda}+\delta_{\lambda x}+\delta_{\lambda x x x}=y_{\lambda} \phi_{\lambda x}, \\
& \delta_{\lambda}(0)=\delta_{\lambda}(L)=0, \quad \delta_{\lambda x}(0)=\delta_{\lambda x}(L) .
\end{aligned}
$$

From 2.39, 2.40, 2.49, 2.72 and 2.73, we get

$$
\frac{d}{d t} \int_{0}^{L} e^{2 \lambda t} \delta_{\lambda} \alpha_{2} d x=e^{2 \lambda t} \delta_{\lambda x}(L)\left(\alpha_{2 x}(t, L)-\alpha_{2 x}(t, 0)\right)+\int_{0}^{L} e^{\lambda t} \phi_{\lambda x} \alpha_{1} \alpha_{2} d x
$$

which, together with 2.41, gives

$$
\int_{0}^{T} \int_{0}^{L} e^{\lambda t} \phi_{\lambda x} \alpha_{1} \alpha_{2} d x d t+\int_{0}^{T} e^{2 \lambda t} \delta_{\lambda x}(L)\left(\alpha_{2 x}(t, L)-\alpha_{2 x}(t, 0)\right) d t=0 .
$$


From 2.46, 2.71) and 2.75, we get

$$
\int_{0}^{T}\left(e^{2 \lambda t}\left(\delta_{\lambda x}(L)-\psi_{\lambda x}(L)\right)\left(\alpha_{2 x}(t, L)-\alpha_{2 x}(t, 0)\right)+\frac{L}{2} \theta_{1} \alpha_{2 x}(t, L)\right) d t=0 .
$$

Let us restrict ourselves to the case where

$$
\alpha_{2}=0 \quad \text { on }[3 T / 4, T] \times[0, L] .
$$

This allows us to perform a time translation of $\epsilon \in[0, T / 4]$ : if we define

$$
\begin{array}{ll}
\alpha_{2 \epsilon}(t, x):=\alpha_{2}(t-\epsilon, x), & \forall t \in[\epsilon, T], \\
\alpha_{2 \epsilon}(t, x):=0, & \forall t \in[0, \epsilon],
\end{array}
$$

then $\alpha_{2 \epsilon}$ also satisfies 2.39), 2.40) (in the mild sense) and 2.41. Hence, by 2.76 associated to $\alpha_{2 \epsilon}$,

$\int_{0}^{T}\left(e^{2 \lambda t}\left(\delta_{\lambda x}(L)-\psi_{\lambda x}(L)\right)\left(\alpha_{2 x}(t-\epsilon, L)-\alpha_{2 x}(t-\epsilon, 0)\right)+\frac{L}{2} \theta_{1} \alpha_{2 x}(t-\epsilon, L)\right) d t=0$ for all $\epsilon \in[0, T / 4]$, which is equivalent to

$$
\int_{0}^{T}\left(e^{2 \lambda(t+\epsilon)}\left(\delta_{\lambda x}(L)-\psi_{\lambda x}(L)\right)\left(\alpha_{2 x}(t, L)-\alpha_{2 x}(t, 0)\right)+\frac{L}{2} \theta_{1} \alpha_{2 x}(t, L)\right) d t=0
$$

for all $\epsilon \in[0, T / 4]$. This last property, together with 2.51], implies that

$$
\left(\delta_{\lambda x}(L)-\psi_{\lambda x}(L)\right) \int_{0}^{T}\left(e^{2 \lambda t}\left(\alpha_{2 x}(t, L)-\alpha_{2 x}(t, 0)\right)\right) d t=0 .
$$

Let $a \in \mathbb{R} \backslash[-1 / \sqrt{3}, 1 / \sqrt{3}]$. We take $\lambda:=2 i a\left(4 a^{2}-1\right)$. Let

$$
y_{\lambda}(x):=\kappa e^{\left(-\sqrt{3 a^{2}-1}-i a\right) x}+(1-\kappa) e^{\left(\sqrt{3 a^{2}-1}-i a\right) x}-e^{2 i a x}
$$

with

$$
\kappa:=\frac{e^{2 i a L}-e^{\left(\sqrt{3 a^{2}-1}-i a\right) L}}{e^{\left(-\sqrt{3 a^{2}-1}-i a\right) L}-e^{\left(\sqrt{3 a^{2}-1}-i a\right) L}} .
$$

One easily checks that such a $y_{\lambda}$ satisfies 2.53, 2.54) and 2.56. Let, with $\lambda:=$ $2 i a\left(4 a^{2}-1\right)$,

$$
\Sigma:=\{a \in \mathbb{R} \backslash[-1 / \sqrt{3}, 1 / \sqrt{3}] ; \lambda \notin \sigma(\tilde{A}) \text { and } 2 \lambda \notin \sigma(\tilde{A})\} .
$$

Then the function $S: \Sigma \rightarrow \mathbb{C}, S(a)=\delta_{\lambda x}(L)-\psi_{\lambda x}(L)$, is continuous (and even analytic). In Appendix C we prove the following lemma:

Lemma 11. The function $S$ is not identically equal to 0 . 
This lemma and (2.80) imply that

$$
\alpha_{2 x}(t, L)-\alpha_{2 x}(t, 0)=0 .
$$

Indeed, let $\mathcal{L}: \mathbb{C} \rightarrow \mathbb{C}, \lambda \mapsto \int_{0}^{T}\left(e^{2 \lambda t}\left(\alpha_{2 x}(t, L)-\alpha_{2 x}(t, 0)\right)\right) d t$. The function $\mathcal{L}$ is holomorphic. Hence the zeros of $\mathcal{L}$ are isolated if $\mathcal{L} \neq 0$. However, by Lemma 11], 2.80, and the continuity of $S$, there exists a nonempty open subset of $i \mathbb{R}$ on which $\mathcal{L}$ vanishes (let us recall that $\sigma(\tilde{A})$ is a discrete subset of $i \mathbb{R}$ ). Hence $\mathcal{L}=0$, which implies 2.83).

We multiply 2.39 by $\bar{\alpha}_{2}$, take the real part, and integrate on $[0, L]$. Then, using integrations by parts and $(2.40)$ together with 2.83$)$, we get

$$
\frac{d}{d t} \int_{0}^{L}\left|\alpha_{2}\right|^{2} d x=0
$$

which, combined with 2.41, implies that

$$
\alpha_{2}=0 .
$$

But, by Theorem 3 (for mild solutions), there are mild solutions of (2.39) and (2.40) satisfying (2.41) and (2.77) such that (2.84) does not hold. This ends the proof of Proposition 10 and therefore of Proposition 8 .

\section{Local exact controllability}

In this section we still assume that 2.5 holds and we end the proof of Theorem 2 As pointed out in Section 11, the invariance of the control system $(\mathrm{KdV})$ under the change of variables $\tau=T-t, \xi=L-x$ allows us to prove only that, for every $T>0$, there exists $r_{1}^{\prime}>0$ such that, for every $y_{T} \in L^{2}(0, L)$ with $\left\|y_{T}\right\|_{L^{2}(0, L)} \leq r_{1}^{\prime}$, there exists $u \in L^{2}(0, T)$ such that the mild solution $y$ of

$$
\begin{aligned}
& y_{t}+y_{x}+y_{x x x}+y y_{x}=0, \\
& y(t, 0)=y(t, L)=0, \\
& y_{x}(t, L)=u(t), \\
& y(0, x)=0,
\end{aligned}
$$

satisfies $y(T, \cdot)=y_{T}$. Of course, by " $y$ is a mild solution of 3.1) to 3.4", we mean that $y$ is in $B$ and is the mild solution of

$$
y_{t}+y_{x}+y_{x x x}=f, \quad y(t, 0)=y(t, L)=0, y_{x}(t, L)=u(t), y(0, x)=0,
$$

with $f:=-y y_{x}$ (note that, if $y$ is in $B$, then $y y_{x} \in L^{1}\left(0, T, L^{2}(0, L)\right)$ ). We use similar natural conventions until the end of this paper. It follows from Propositions 14 and 15 below that, for a given $u \in L^{2}(0, T)$, there exists at most one mild solution of 3.1) to 3.4 and that such a solution exists if $\|u\|_{L^{2}(0, T)}$ is small enough (the smallness depending on $T$ and $L$ ). 
By (the proof of) Theorem 3 , there exists a continuous linear map

$$
\Gamma: h \in H \subset L^{2}(0, L) \mapsto \Gamma(h) \in L^{2}(0, T)
$$

such that the mild solution of

$$
y_{t}+y_{x}+y_{x x x}=0, \quad y(t, 0)=y(t, L)=0, y_{x}(t, L)=\Gamma(h)(t), y(0, x)=0,
$$

satisfies $y(T, x)=h(x)$. (One can take for $\Gamma$ the control obtained by means of HUM; see [12, Remark 3.10].)

Let $y_{T} \in L^{2}(0, L)$ be such that $\left\|y_{T}\right\|_{L^{2}(0, L)} \leq r, r>0$ to be chosen later, small enough so that the maps introduced below are well defined in a neighborhood of 0 . Let $\mathcal{T}_{y_{T}}$ denote the map

$$
\mathcal{T}_{y_{T}}: L^{2}(0, L) \rightarrow L^{2}(0, L), \quad z \mapsto z+y_{T}-F(G(z)),
$$

with

$$
F: L^{2}(0, T) \rightarrow L^{2}(0, L), \quad u \mapsto y(T, \cdot),
$$

where $y$ is the mild solution of 3.1 to 3.4 and $G: L^{2}(0, L) \rightarrow L^{2}(0, T)$ is defined as follows. We decompose $z=\mathcal{P}_{H}(z)+\rho(z)(1-\cos (x))$. Then

1. If $\rho(z) \geq 0$, then $G(z)=\Gamma\left(\mathcal{P}_{H}(z)\right)+\rho^{1 / 3}(z) u_{+}+\rho^{2 / 3}(z) v_{+}+\rho(z) w_{+}$.

2. If $\rho(z)<0$, then $G(z)=\Gamma\left(\mathcal{P}_{H}(z)\right)+|\rho(z)|^{1 / 3} u_{-}+|\rho(z)|^{2 / 3} v_{-}+|\rho(z)| w_{-}$.

(The functions $u_{ \pm}, v_{ \pm}$and $w_{ \pm}$are fixed as in Proposition 8 )

Clearly, each fixed point $z^{*}$ of $\mathcal{T}_{y_{T}}$ satisfies $F\left(G\left(z^{*}\right)\right)=y_{T}$, and the control $u=$ $G\left(z^{*}\right)$ is a solution to our problem.

Until the end of this paper, we adopt the following notations:

- For $z \in L^{2}\left(0, T, H^{1}(0, L)\right),\|z\|_{L^{2}\left(H^{1}\right)}=\|z\|_{L^{2}\left(0, T, H^{1}(0, L)\right)}$,

- For $z \in L^{1}\left(0, T, L^{2}(0, L)\right),\|z\|_{L^{1}\left(L^{2}\right)}=\|z\|_{L^{1}\left(0, T, L^{2}(0, L)\right)}$,

- $\mathcal{B}_{R}=\left\{z \in L^{2}(0, L) ;\|z\|_{L^{2}(0, L)} \leq R\right\}$.

First of all we prove a lemma about the map $\mathcal{T}_{0}$.

Lemma 12. There exist $C_{1}=C_{1}(T, L)>0$ and $\epsilon_{1}=\epsilon_{1}(T, L)>0$ such that, for every $z \in \mathcal{B}_{\epsilon_{1}}$,

$$
\left\|\mathcal{T}_{0} z\right\|_{L^{2}(0, L)} \leq C_{1}\|z\|_{L^{2}(0, L)}^{4 / 3} .
$$

Let $z \in L^{2}(0, L)$. Let $(\tilde{u}, \tilde{v}, \tilde{w})=\left(u_{+}, v_{+}, w_{+}\right)$if $\rho(z) \geq 0$ and $(\tilde{u}, \tilde{v}, \tilde{w})=\left(u_{-}, v_{-}, w_{-}\right)$ if $\rho(z)<0$. Let $y$ be the mild solution of

$$
\begin{aligned}
& y_{t}+y_{x}+y_{x x x}+y y_{x}=0 \\
& y(t, 0)=y(t, L)=0 \\
& y_{x}(t, L)=\Gamma\left(\mathcal{P}_{H}(z)\right)(t)+|\rho(z)|^{1 / 3} \tilde{u}(t)+|\rho(z)|^{2 / 3} \tilde{v}(t)+|\rho(z)| \tilde{w}(t), \\
& y(0, x)=0
\end{aligned}
$$


By Propositions 14 and 15, there exist $\epsilon_{2}=\epsilon_{2}(T, L)>0$ and $C_{2}=C_{2}(T, L)>0$ such that, for every $z \in L^{2}(0, L)$ with $\|z\|_{L^{2}(0, L)} \leq \epsilon_{2}$, there exists a unique mild solution of (3.7) to 3.10, and this mild solution satisfies

$$
\|y\|_{B} \leq C_{2}\|z\|_{L^{2}(0, L)}^{1 / 3} .
$$

Let $\tilde{y}, \tilde{\alpha}, \tilde{\beta}, \tilde{\gamma}$ be the mild solutions of

$$
\begin{aligned}
& \tilde{y}_{t}+\tilde{y}_{x}+\tilde{y}_{x x x}=0, \\
& \tilde{y}(t, 0)=\tilde{y}(t, L)=0, \\
& \tilde{y}_{x}(t, L)=\Gamma\left(\mathcal{P}_{H}(z)\right)(t), \\
& \tilde{y}(0, x)=0, \\
& \tilde{\alpha}_{t}+\tilde{\alpha}_{x}+\tilde{\alpha}_{x x x}=0, \\
& \tilde{\alpha}(t, 0)=\tilde{\alpha}(t, L)=0, \\
& \tilde{\alpha}_{x}(t, L)=\tilde{u}(t), \\
& \tilde{\alpha}(0, x)=0, \\
& 2 \tilde{\beta}_{t}+\tilde{\beta}_{x}+\tilde{\beta}_{x x x}=-\tilde{\alpha} \tilde{\alpha}_{x}, \\
& \tilde{\beta}(t, 0)=\tilde{\beta}(t, L)=0, \\
& \tilde{\beta}_{x}(t, L)=\tilde{v}(t), \\
& \tilde{\beta}(0, x)=0, \\
& \tilde{\gamma} t+\tilde{\gamma}_{x}+\tilde{\gamma}_{x x x}=-(\tilde{\alpha} \tilde{\beta})_{x}, \\
& \tilde{\gamma}(t, 0)=\tilde{\gamma}(t, L)=0, \\
& \tilde{\gamma}(t, L)=\tilde{w}(t), \\
& \tilde{\gamma}(0, x)=0 .
\end{aligned}
$$

Let

$$
\begin{aligned}
\phi:= & y-\tilde{y}-|\rho(z)|^{1 / 3} \tilde{\alpha}-|\rho(z)|^{2 / 3} \tilde{\beta}-|\rho(z)| \tilde{\gamma}, \\
a:= & \tilde{y}+|\rho(z)|^{1 / 3} \tilde{\alpha}+|\rho(z)|^{2 / 3} \tilde{\beta}+|\rho(z)| \tilde{\gamma}, \\
b:= & \tilde{y} \tilde{y}_{x}+\left(\tilde{y}\left(|\rho(z)|^{1 / 3} \tilde{\alpha}+|\rho(z)|^{2 / 3} \tilde{\beta}+|\rho(z)| \tilde{\gamma}\right)\right)_{x} \\
& +|\rho(z)|^{4 / 3}(\tilde{\alpha} \tilde{\gamma})_{x}+|\rho(z)|^{4 / 3} \tilde{\beta} \tilde{\beta}_{x}+|\rho(z)|^{5 / 3}(\tilde{\beta} \tilde{\gamma})_{x}+|\rho(z)|^{2} \tilde{\gamma} \tilde{\gamma}_{x} .
\end{aligned}
$$

By Proposition 7, (3.11) to (3.30) and standard estimates, there exists $C_{3}=C_{3}(T, L)>0$ such that, for every $z \in L^{2}(0, L)$ with $\|z\|_{L^{2}(0, L)} \leq \epsilon_{2}$,

$$
\begin{aligned}
\|a\|_{B} & \leq C_{3}\|z\|_{L^{2}(0, L)}^{1 / 3}, \\
\|b\|_{L^{1}\left(L^{2}\right)} & \leq C_{3}\|z\|_{L^{2}(0, L)}^{4 / 3}, \\
\|\phi\|_{B} & \leq C_{3}\|z\|_{L^{2}(0, L)}^{1 / 3}
\end{aligned}
$$


Similarly standard estimates give the existence of $C_{4}=C_{4}(T, L)>0$ such that

$$
\left\|(\phi a)_{x}\right\|_{L^{1}\left(L^{2}\right)} \leq C_{4}\|\phi\|_{B}\|a\|_{B} .
$$

Note that, by 2.18, 3.7 to 3.10 and 3.12 to 3.28,

$$
\phi(T, \cdot)=F(G(z))-z=-\mathcal{T}_{0} z
$$

Moreover, by 3.7 to 3.10 and 3.12 to 3.30, $\phi$ is a mild solution of

$$
\begin{aligned}
& \phi_{t}+\phi_{x}+\phi_{x x x}+\phi \phi_{x}=-(\phi a)_{x}-b \\
& \phi(t, 0)=\phi(t, L)=\phi_{x}(t, L)=0 \\
& \phi(0, x)=0
\end{aligned}
$$

From 3.32, 3.34 to 3.37, 3.38 and Proposition 15 there exists $C_{5}=C_{5}(T, L)>0$ such that

$$
\|\phi\|_{B}^{2} \leq\left(\|z\|_{L^{2}(0, L)}^{8 / 3}+\|\phi\|_{B}^{2}\|a\|_{B}^{2}\right) e^{C_{5}\left(1+\|\phi\|_{B}^{2}\right)} .
$$

From 3.31, 3.33 and 3.39, one gets the existence of $\epsilon_{3}=\epsilon_{3}(T, L)>0$ and of $C_{6}=C_{6}(T, L)>0$ such that, for every $z \in L^{2}(0, L)$ with $\|z\|_{L^{2}(0, L)} \leq \epsilon_{3}$,

$$
\|\phi\|_{B} \leq C_{6}\|z\|_{L^{2}(0, L)}^{4 / 3}
$$

which, together with 3.35), ends the proof of Lemma 12

We now study $\mathcal{P}_{H} \circ \mathcal{T}_{y_{T}}$ on the space $H$. For $\omega \in \mathbb{R}$ and $y_{T} \in L^{2}(0, L)$, let

$$
\Pi: H \rightarrow H, \quad g \mapsto g+\mathcal{P}_{H}\left(y_{T}\right)-\mathcal{P}_{H}(F(G(g+\omega e))),
$$

where $e(x):=1-\cos (x)$ and $\omega \in \mathbb{R}$. (In fact we should write, for example, $\Pi_{y_{T}, \omega}$, but for simplicity we omit the indices $y_{T}$ and $\omega$.) To prove the existence of a fixed point for $\Pi$, we apply the Banach fixed point theorem to the restriction of $\Pi$ to the closed ball $\mathcal{B}_{R} \cap H$, with $\left\|y_{T}\right\|_{L^{2}(0, L)}+|\omega| \leq R / 3$ and where $R>0$ small enough. Let $\left(y_{T}, \omega\right) \in L^{2}(0, L) \times \mathbb{R}$ be such that $\left\|y_{T}\right\|_{L^{2}(0, L)}+|\omega| \leq R / 3$. Let $g, h \in H \cap \mathcal{B}_{R}$. With 3.6, we have, for $R>0$ small enough,

$$
\begin{aligned}
\|\Pi(g)\|_{L^{2}(0, L)} & \leq\left\|y_{T}\right\|_{L^{2}(0, L)}+\|g+\omega e-F(G(g+\omega e))\|_{L^{2}(0, L)} \\
& \leq R / 3+2 R / 3=R .
\end{aligned}
$$

Hence, for $R>0$ small enough,

$$
\Pi\left(\mathcal{B}_{R} \cap H\right) \subset \mathcal{B}_{R} \cap H .
$$


Let us now look at the contracting property of $\Pi$. Let $\omega \in \mathbb{R}, g \in H$ and $h \in H$. Let

$$
(\tilde{u}, \tilde{v}, \tilde{w})= \begin{cases}\left(u_{+}, v_{+}, w_{+}\right) & \text {if } \omega \geq 0 \\ \left(u_{-}, v_{-}, w_{-}\right) & \text {if } \omega<0\end{cases}
$$

Let $y, z, \tilde{y}, \tilde{z}$ be the mild solutions of the following problems:

$$
\begin{aligned}
& y_{t}+y_{x}+y_{x x x}+y y_{x}=0, \\
& y(t, 0)=y(t, L)=0, \\
& y_{x}(t, L)=\Gamma(g)(t)+|\omega|^{1 / 3} \tilde{u}(t)+|\omega|^{2 / 3} \tilde{v}(t)+|\omega| \tilde{w}(t), \\
& y(0, x)=0, \\
& z_{t}+z_{x}+z_{x x x}+z z_{x}=0, \\
& z(t, 0)=z(t, L)=0, \\
& z_{x}(t, L)=\Gamma(h)(t)+\omega^{1 / 3} \tilde{u}(t)+\omega^{2 / 3} \tilde{v}(t)+\omega \tilde{w}(t), \\
& z(0, x)=0, \\
& \tilde{y}_{t}+\tilde{y}_{x}+\tilde{y}_{x x x}=0, \\
& \tilde{y}(t, 0)=\tilde{y}(t, L)=0, \\
& \tilde{y}_{x}(t, L)=\Gamma(g)(t), \\
& \tilde{y}(0, x)=0, \\
& \tilde{z}_{t}+\tilde{z}_{x}+\tilde{z}_{x x x}=0, \\
& \tilde{z}(t, 0)=\tilde{z}_{(t, L)=0,}, \\
& \tilde{z}_{x}(t, L)=\Gamma(h)(t), \\
& \tilde{z}(0, x)=0 .
\end{aligned}
$$

Let $\phi=y-\tilde{y}$ and $\psi=z-\tilde{z}$. Let $\gamma=\phi-\psi$. By (3.43) to 3.58, $\gamma$ is a mild solution of

$$
\begin{aligned}
& \gamma_{t}+\gamma_{x}+\gamma_{x x x}+\gamma \gamma_{x}=-(\gamma a)_{x}-b, \\
& \gamma(t, 0)=\gamma(t, L)=0, \quad \gamma_{x}(t, L)=0, \quad \gamma(0, x)=0,
\end{aligned}
$$

with

$$
a=\psi+\tilde{y}, \quad b=(\psi(\tilde{y}-\tilde{z}))_{x}+\tilde{y} \tilde{y}_{x}-\tilde{z} \tilde{z}_{x} .
$$

Let us notice that there exists $C_{7}=C_{7}(T)>0$ such that

$$
\begin{gathered}
\|b\|_{L^{1}\left(L^{2}\right)} \leq C_{7}\left(\|z\|_{B}+\|\tilde{y}\|_{B}+\|\tilde{z}\|_{B}\right)\|\tilde{y}-\tilde{z}\|_{B}, \\
\left\|(\gamma a)_{x}\right\|_{L^{1}\left(L^{2}\right)} \leq C_{7}\left(\|z\|_{B}+\|\tilde{z}\|_{B}+\|\tilde{y}\|_{B}\right)\|\gamma\|_{B} .
\end{gathered}
$$


Then, using again Propositions 14 and 15 as in the the proof of 3.40 , we get the existence of $\epsilon_{4}=\epsilon_{4}(T, L)>0$ such that, for every $(g, h, \omega) \in H \times H \times \mathbb{R}$ with

$$
\|g\|_{L^{2}(0, L)} \leq \epsilon_{4}, \quad\|h\|_{L^{2}(0, L)} \leq \epsilon_{4}, \quad|\omega| \leq \epsilon_{4},
$$

one has

$$
\|\gamma\|_{B} \leq \frac{1}{2}\|g-h\|_{L^{2}(0, L)} .
$$

Note that 3.59 implies that

$$
\|\Pi(g)-\Pi(h)\|_{L^{2}(0, L)}=\|\gamma(T, \cdot)\|_{L^{2}(0, L)} \leq\|\gamma\|_{B} \leq 1 / 2\|g-h\|_{L^{2}(0, L)} .
$$

Therefore, by 3.42 and 3.60 , there exists $\epsilon_{5}=\epsilon_{5}(T, L)>0$ such that, for $R \leq \epsilon_{5}$, for every $\left(y_{T}, \omega\right) \in L^{2}(0, L) \times \mathbb{R}$ such that $\left\|y_{T}\right\|_{L^{2}(0, L)}+|\omega| \leq R / 3$, $\Pi$ has a unique fixed point $h\left(y_{T}, \omega\right)$ in $\mathcal{B}_{R} \cap H$. Note that the map $h$ is continuous in a neighborhood of $(0,0) \in H \times \mathbb{R}$.

We now apply the intermediate value theorem to the map

$$
\tau: \mathbb{R} \rightarrow \mathbb{R}, \quad \omega \mapsto \rho\left(\omega e+h\left(y_{T}, \omega\right)+y_{T}-F\left(G\left(h\left(y_{T}, \omega\right)+\omega e\right)\right)\right) .
$$

By (3.6), there exists $\epsilon_{6}=\epsilon_{6}(T, L)>0$ such that, if $\left\|y_{T}\right\|_{L^{2}(0, L)} \leq \epsilon_{6} \sqrt{3 L / 8}$, then $\tau\left(\left[-\epsilon_{6}, \epsilon_{6}\right]\right) \subset\left[-\epsilon_{6}, \epsilon_{6}\right]$. Hence, if $\left\|y_{T}\right\|_{L^{2}(0, L)} \leq \epsilon_{6} \sqrt{3 L / 8}$, we deduce, by the intermediate value theorem, that $\tau$ has at least one fixed point $\omega_{0}$. We have

$$
F\left(G\left(h\left(y_{T}, \omega_{0}\right)+\omega_{0} e\right)\right)=y_{T},
$$

which ends the proof of Theorem 2.

Let us remark that it follows from our proof of Theorem 2 that the following theorem, slightly more precise than Theorem 2 , also holds.

Theorem 13. Let $k$ be a positive integer and let $T>0$. There exist $r_{1}>0$ and $C>0$ such that, for every $\left(y_{0}, y_{T}\right) \in L^{2}(0,2 k \pi)^{2}$ with $\left\|y_{0}\right\|_{L^{2}(0,2 k \pi)}<r_{1}$ and $\left\|y_{T}\right\|_{L^{2}(0,2 k \pi)}$ $<r_{1}$, there exists $y \in C\left([0, T], L^{2}(0,2 k \pi)\right) \cap L^{2}\left(0, T, H^{1}(0,2 k \pi)\right)$ satisfying, in the mild sense, $(\mathrm{KdV})$ with $L=2 k \pi$ such that

$$
\begin{gathered}
y(0, \cdot)=y_{0}, \quad y(T, \cdot)=y_{T}, \\
\|y\|_{B} \leq C\left(\left\|\mathcal{P}_{H}\left(y_{0}\right)\right\|_{L^{2}(0,2 k \pi)}+\left|\int_{0}^{2 k \pi}(1-\cos (x)) y_{0} d x\right|^{1 / 3}\right. \\
\left.+\left\|\mathcal{P}_{H}\left(y_{1}\right)\right\|_{L^{2}(0,2 k \pi)}+\left|\int_{0}^{2 k \pi}(1-\cos (x)) y_{1} d x\right|^{1 / 3}\right) .
\end{gathered}
$$




\section{A. Appendix: Existence and uniqueness of solutions to Cauchy problems for KdV equations}

We first prove the existence of solutions to the Cauchy problem for nonlinear KdV equations (with small data).

Proposition 14. Let $L>0$ and $T>0$. There exist $\epsilon>0$ and $C>0$ such that, for every $f \in L^{1}\left(0, T, L^{2}(0, L)\right)$, every $u \in L^{2}(0, T)$ and every $y_{0} \in L^{2}(0, L)$ such that

$$
\|f\|_{L^{1}\left(L^{2}\right)}+\|u\|_{L^{2}(0, T)}+\left\|y_{0}\right\|_{L^{2}(0, L)} \leq \epsilon,
$$

there exists at least one mild solution y of

$$
\left\{\begin{array}{l}
y_{t}+y_{x}+y_{x x x}+y y_{x}=f \\
y(t, 0)=y(t, L)=0 \\
y_{x}(t, L)=u(t) \\
y(0, x)=y_{0}(x)
\end{array}\right.
$$

which satisfies

$$
\|y\|_{B} \leq C\left(\|f\|_{L^{1}\left(L^{2}\right)}+\|u\|_{L^{2}(0, T)}+\left\|y_{0}\right\|_{L^{2}(0, L)}\right) .
$$

Proof. Let $T>0$. For $f \in L^{1}\left(0, T, L^{2}(0, L)\right), u \in L^{2}(0, T)$ and $y_{0} \in L^{2}(0, L)$, define

$$
M_{f, u, y_{0}}: B \rightarrow B, \quad y \mapsto \Psi\left(y_{0}, u, f-y y_{x}\right) .
$$

A fixed point of $M_{f, u, y_{0}}$ is a solution of A.1 . One easily gets the existence of $C_{8}=$ $C_{8}(T)>0$ such that, for every $(y, z) \in B^{2}$,

$$
\begin{aligned}
\left\|y y_{x}\right\|_{L^{1}\left(L^{2}\right)} & \leq C_{8}\|y\|_{B}^{2}, \\
\left\|z z_{x}-y y_{x}\right\|_{L^{1}\left(L^{2}\right)} & \leq C_{8}\left(\|z\|_{B}+\|y\|_{B}\right)\|z-y\|_{B} .
\end{aligned}
$$

Hence, by continuity of $\Psi$ (see Proposition 77 , there exists a constant $C_{9}=C_{9}(T, L)>0$ such that, for every $f \in L^{1}\left(0, T, L^{2}(0, L)\right)$, every $u \in L^{2}(0, T)$, every $y_{0} \in L^{2}(0, L)$, every $y$ in $B$ and every $z$ in $B$,

$$
\begin{gathered}
\left\|M_{f, u, y_{0}}(y)\right\|_{B} \leq C_{9}\left(\|f\|_{L^{1}\left(L^{2}\right)}+\|u\|_{L^{2}(0, T)}+\left\|y_{0}\right\|_{L^{2}(0, L)}+\|y\|_{B}^{2}\right), \\
\left\|M_{f, u, y_{0}}(z)-M_{f, u, y_{0}}(y)\right\|_{B} \leq C_{9}\left(\|z\|_{B}+\|y\|_{B}\right)\|z-y\|_{B} .
\end{gathered}
$$

From these two inequalities and the Banach fixed point theorem, one sees that the assertion of Proposition 14 holds with

$$
\epsilon:=\frac{1}{9 C_{9}^{2}}, \quad C:=\frac{3 C_{9}}{2} .
$$

(Note that $M_{f, u, y_{0}}(\tilde{B}) \subset \tilde{B}$ if $\tilde{B}:=\left\{y \in B ;\|y\|_{B} \leq 1 /\left(3 C_{9}\right)\right\},\left\|M_{f, u, y_{0}}(z)-M_{f, u, y_{0}}(y)\right\|_{B}$ $\leq(2 / 3)\|z-y\|_{B}$ for every $(y, z) \in \tilde{B}^{2}$, and $\|y\|_{L^{2}(0, L)}\left(1-C_{9}\|y\|_{L^{2}(0, L)}\right) \leq C_{9}\left(\|f\|_{L^{1}\left(L^{2}\right)}\right.$ $\left.+\|u\|_{L^{2}(0, T)}+\left\|y_{0}\right\|_{L^{2}(0, L)}\right)$ if $y$ is a fixed point of $M_{f, u, y_{0}}$.)

We now prove the uniqueness of the mild solution of the Cauchy problem for our nonlinear $\mathrm{KdV}$ equation, together with estimates of this solution. 
Proposition 15. Let $T>0$ and let $L>0$. There exists $C_{10}=C_{10}(T, L)>0$ such that for every $\left(y_{0}, z_{0}\right) \in L^{2}(0, L)^{2},(u, v) \in L^{2}(0, T)^{2}$ and $(f, g) \in L^{1}\left(0, T, L^{2}(0, L)\right)^{2}$ for which there exist mild solutions $y$ and $z$ of

$$
\begin{aligned}
& y_{t}+y_{x}+y_{x x x}+y y_{x}=f, \\
& y(t, 0)=y(t, L)=0, \\
& y_{x}(t, L)=u(t), \\
& y(0, x)=y_{0}(x),
\end{aligned}
$$

and of

$$
\begin{aligned}
& z_{t}+z_{x}+z_{x x x}+z z_{x}=g, \\
& z(t, 0)=z(t, L)=0, \\
& z_{x}(t, L)=v(t), \\
& z(0, x)=z_{0}(x),
\end{aligned}
$$

one has the following inequalities:

$$
\begin{aligned}
& \int_{0}^{T} \int_{0}^{L}\left(z_{x}(t, x)\right.\left.-y_{x}(t, x)\right)^{2} d x d t \\
& \leq\left(\int_{0}^{L}\left(z_{0}-y_{0}\right)^{2} d x+\|u-v\|_{L^{2}(0, T)}^{2}+\|f-g\|_{L^{1}\left(L^{2}\right)}^{2}\right) \\
& \cdot e^{C_{10}\left(1+\|y\|_{L^{2}\left(H^{1}\right)}^{2}+\|z\|_{L^{2}\left(H^{1}\right)}^{2}\right)}, \\
& \int_{0}^{L}(z(t, x)-y(t, x))^{2} d x \\
& \leq\left(\int_{0}^{L}\left(z_{0}-y_{0}\right)^{2} d x+\|u-v\|_{L^{2}(0, T)}^{2}+\|f-g\|_{L^{1}\left(L^{2}\right)}^{2}\right) \\
& \cdot e^{C_{10}\left(1+\|y\|_{L^{2}\left(H^{1}\right)}^{2}+\|z\|_{L^{2}\left(H^{1}\right)}^{2}\right)},
\end{aligned}
$$

for all $t \in[0, T]$.

\section{Proof. Let}

$$
\Delta:=z-y .
$$

Then $\Delta$ is a mild solution of

$$
\begin{aligned}
& \Delta_{t}+\Delta_{x}+\Delta_{x x x}=-y \Delta_{x}-z_{x} \Delta-(f-g), \\
& \Delta(t, 0)=\Delta(t, L)=0 \\
& \Delta_{x}(t, L)=v(t)-u(t) \\
& \Delta(0, x)=z_{0}(x)-y_{0}(x) .
\end{aligned}
$$


386

Jean-Michel Coon, Emmanuelle Crépeau

Formally, by integrating by parts in

$$
\int_{0}^{L} 2 x \Delta\left(\Delta_{t}+\Delta_{x}+\Delta_{x x x}+y \Delta_{x}+z_{x} \Delta+(f-g)\right) d x=0,
$$

using A.14 and A.15, we readily get

$$
\begin{aligned}
\frac{d}{d t} \int_{0}^{L} x \Delta^{2} d x+3 \int_{0}^{L} \Delta_{x}^{2}= & \int_{0}^{L} \Delta^{2} d x+L(v(t)-u(t))^{2}-2 \int_{0}^{L} x y \Delta \Delta_{x} d x \\
& +2 \int_{0}^{L} z \Delta^{2} d x+4 \int_{0}^{L} x z \Delta \Delta_{x} d x \\
& -2 \int_{0}^{L} x \Delta(f-g) d x
\end{aligned}
$$

(Note that the multiplier $x \Delta$ for A.13 has been introduced by Rosier in [12, p. 48].) Even if A.17) is only formal, using standard approximation arguments, one easily sees that A.18) always holds (in the sense of distributions).

By $\left[\right.$ A.3 and the continuous Sobolev embedding $H_{0}^{1}(0, L) \subset C^{0}([0, T])$, there exists $C_{11}=C_{11}(L)>0$ such that

$$
2\left|\int_{0}^{L} x y \Delta \Delta_{x} d x\right| \leq C_{11}\left\|y_{x}\right\|_{L^{2}(0, L)} \int_{0}^{L}\left|x \Delta \Delta_{x}\right| d x .
$$

Thus,

$$
2\left|\int_{0}^{L} x y \Delta \Delta_{x} d x\right| \leq \frac{1}{2} \int_{0}^{L} \Delta_{x}^{2} d x+\frac{C_{11}^{2}}{2}\left\|y_{x}\right\|_{L^{2}(0, L)}^{2} L \int_{0}^{L} x \Delta^{2} d x .
$$

Similarly,

$$
4\left|\int_{0}^{L} x z \Delta \Delta_{x} d x\right| \leq \frac{1}{2} \int_{0}^{L} \Delta_{x}^{2} d x+2 C_{11}^{2}\left\|z_{x}\right\|_{L^{2}(0, L)}^{2} \int_{0}^{L} x \Delta^{2} d x .
$$

We have the following lemma:

Lemma 16. For every $\phi \in H^{1}(0, L)$ with $\phi(0)=0$, and every a $\in[0, L]$,

$$
\int_{0}^{L} \phi^{2} d x \leq \frac{a^{2}}{2} \int_{0}^{L} \phi_{x}^{2} d x+\frac{1}{a} \int_{0}^{L} x \phi^{2} d x .
$$

Indeed,

$$
\begin{aligned}
\int_{0}^{L} \phi^{2} d x & =\int_{0}^{a} \phi^{2} d x+\int_{a}^{L} \phi^{2} d x \leq \int_{0}^{a}\left(\int_{0}^{x} \phi_{x}(s) d s\right)^{2} d x+\frac{1}{a} \int_{0}^{L} x \phi^{2} d x \\
& \leq \int_{0}^{a}\left(\int_{0}^{L} \phi_{x}^{2}(s) d s\right) x d x+\frac{1}{a} \int_{0}^{L} x \phi^{2} d x \\
& \leq \frac{a^{2}}{2} \int_{0}^{L} \phi_{x}^{2} d x+\frac{1}{a} \int_{0}^{L} x \phi^{2} d x .
\end{aligned}
$$


Thanks to Lemma 16, there exists $C_{12}>0$ such that

$$
\int_{0}^{L} \Delta^{2} d x \leq \frac{1}{2} \int_{0}^{L} \Delta_{x}^{2} d x+C_{12} \int_{0}^{L} x \Delta^{2} d x .
$$

Moreover, by A.7) and the Sobolev embedding $H_{0}^{1}(0, L) \subset C^{0}([0, T])$, there exists $C_{13}=C_{13}(L)>0$ such that

$$
2\left|\int_{0}^{L} z \Delta^{2} d x\right| \leq C_{13}\left\|z_{x}\right\|_{L^{2}(0, L)} \int_{0}^{L} \Delta^{2} d x .
$$

Hence, using A.14) and Lemma 16 with $a:=\min \left\{C_{13}^{-1 / 2}\left\|z_{x}\right\|_{L^{2}(0, L)}^{-1 / 2}, L\right\}$, there exists $C_{14}=C_{14}(L)>0$ such that

$$
2 \int_{0}^{L} z \Delta^{2} d x \leq \frac{1}{2} \int_{0}^{L} \Delta_{x}^{2} d x+C_{14}\left(1+\left\|z_{x}\right\|_{L^{2}(0, L)}^{3 / 2}\right) \int_{0}^{L} x \Delta^{2} d x .
$$

Moreover,

$$
2\left|\int_{0}^{L} x \Delta(f-g) d x\right| \leq 2 \sqrt{L}\|f-g\|_{L^{2}(0, L)}\left(\int_{0}^{L} x \Delta^{2} d x\right)^{1 / 2} .
$$

Thus, by A.20, A.22, A.23, and A.24, there exists $C_{15}=C_{15}(L)>0$ such that

$$
\begin{aligned}
\frac{d}{d t} \int_{0}^{L} x \Delta^{2} d x & +\int_{0}^{L} \Delta_{x}^{2} d x \\
\leq & L(v(t)-u(t))^{2}+2 \sqrt{L}\|f-g\|_{L^{2}(0, L)}\left(\int_{0}^{L} x \Delta^{2} d x\right)^{1 / 2} \\
& +C_{15}\left(1+\left\|y_{x}\right\|_{L^{2}(0, L)}^{2}+\left\|z_{x}\right\|_{L^{2}(0, L)}^{2}\right) \int_{0}^{L} x \Delta^{2} d x
\end{aligned}
$$

In particular,

$$
\begin{aligned}
\frac{d}{d t} \int_{0}^{L} x \Delta^{2} d x \leq & L(v(t)-u(t))^{2}+2 \sqrt{L}\|f-g\|_{L^{2}(0, L)}\left(\int_{0}^{L} x \Delta^{2} d x\right)^{1 / 2} \\
& +C_{15}\left(1+\left\|y_{x}\right\|_{L^{2}(0, L)}^{2}+\left\|z_{x}\right\|_{L^{2}(0, L)}^{2}\right) \int_{0}^{L} x \Delta^{2} d x
\end{aligned}
$$

Let us assume for the time being that the following lemma holds:

Lemma 17. Let $T>0$. Let $a, b$ and $c$ be three nonnegative functions in $L^{1}(0, T)$. Let $w \in C^{0}([0, T])$ be a nonnegative function such that, in the sense of distributions,

$$
\dot{w} \leq a(t)+b(t) \sqrt{w(t)}+c(t) w(t) .
$$

Then

$$
w(t) \leq 3\left(w(0)+\int_{0}^{t} a(s) d s+\left(\int_{0}^{t} b(s) d s\right)^{2}\right) e^{\int_{0}^{t} c(s) d s} .
$$


From A.16, A.26 and Lemma 17, we get, for every $t \in[0, T]$,

$$
\begin{aligned}
\int_{0}^{L} x \Delta^{2}(t, x) d x \leq & 3\left(\int_{0}^{L} x\left(z 0-y_{0}\right)^{2} d x+L\|u-v\|_{L^{2}(0, T)}^{2}+4 L\|f-g\|_{L^{1}\left(L^{2}\right)}^{2}\right) \\
& \cdot e^{C_{15}\left(T+\|y\|_{L^{2}\left(H^{1}\right)}^{2}+\|z\|_{L^{2}\left(H^{1}\right)}^{2}\right)} .
\end{aligned}
$$

Using A.12, A.25 and A.27, we get the existence of $C_{16}=C_{16}(T, L)>0$ such that

$$
\begin{aligned}
\int_{0}^{T} \int_{0}^{L}\left(z_{x}(t, x)\right. & \left.-y_{x}(t, x)\right)^{2} d x d t \\
\leq & \left(\int_{0}^{L}\left(z_{0}-y_{0}\right)^{2} d x+\|u-v\|_{L^{2}(0, T)}^{2}+\|f-g\|_{L^{1}\left(L^{2}\right)}^{2}\right) \\
\cdot & e^{C_{16}\left(1+\|y\|_{L^{2}\left(H^{1}\right)}^{2}+\|z\|_{L^{2}\left(H^{1}\right)}^{2}\right)},
\end{aligned}
$$

which gives A.10]. Finally, in order to get A.11, we multiply A.13 by $\Delta$ and integrate on $[0, L]$. Using A.14, A.15 and integrations by parts, we get

$$
\begin{aligned}
\frac{1}{2} \frac{d}{d t} \int_{0}^{L} \Delta^{2} d x+\frac{1}{2} \Delta_{x}^{2}(t, 0)= & \frac{1}{2}(v(t)-u(t))^{2} \\
& -\int_{0}^{L}\left(y \Delta_{x}-2 z \Delta_{x}+f-g\right) \Delta d x .
\end{aligned}
$$

Moreover

$$
-\int_{0}^{L}\left(y \Delta_{x}-2 z \Delta_{x}\right) \Delta d x \leq \int_{0}^{L} \Delta_{x}^{2} d x+\int_{0}^{L}\left(\frac{1}{2} y^{2}+2 z^{2}\right) \Delta^{2} d x .
$$

By A.3, A.7, A.29, A.30, and the continuous Sobolev embedding $H_{0}^{1}(0, L) \subset$ $C^{0}([0, L])$ there exists $C_{17}=C_{17}(L)>0$ such that

$$
\begin{aligned}
\frac{1}{2} \frac{d}{d t} \int_{0}^{L} \Delta^{2} d x \leq & \frac{1}{2}(v(t)-u(t))^{2}+\int_{0}^{L} \Delta_{x}^{2} d x \\
& +\|f(t, \cdot)-g(t, \cdot)\|_{L^{2}(0, L)}\left(\int_{0}^{L} \Delta^{2} d x\right)^{1 / 2} \\
& +C_{17}\left(\left\|y_{x}\right\|_{L^{2}(0, L)}^{2}+\left\|z_{x}\right\|_{L^{2}(0, L)}^{2}\right) \int_{0}^{L} \Delta^{2} d x
\end{aligned}
$$

which, combined with A.16, A.28 and Lemma 17, gives A.11 for $C_{10}=C_{10}(T, L)$ $>0$ large enough.

It remains to prove Lemma 17 Considering

$$
\tilde{w}(t):=w(t) e^{-\int_{0}^{t} c(s) d s},
$$


we easily see that, without loss of generality, we may assume that $c=0$. Moreover, we may also assume that there exists $\epsilon>0$ such that, for almost every $s$ in $[0, T], a(s) \geq \epsilon$. Let

$$
\tilde{w}(t):=\max \left(w(t)-\int_{0}^{t} a(s) d s, 0\right) .
$$

Then, in the sense of distributions,

$$
\frac{d}{d t}\left(\sqrt{\tilde{w}(t)+\int_{0}^{t} a(s) d s}\right) \leq \frac{b(t)}{2}+\frac{a(t)}{2 \sqrt{\int_{0}^{t} a(s) d s}} .
$$

Integrating this inequality on $[0, t]$, we get

$$
\sqrt{\tilde{w}(t)+\int_{0}^{t} a(s) d s} \leq \sqrt{w(0)}+\frac{1}{2} \int_{0}^{t} b(s) d s+\sqrt{\int_{0}^{t} a(s) d s},
$$

which gives

$$
w(t) \leq 3\left(w(0)+\frac{1}{4}\left(\int_{0}^{t} b(s) d s\right)^{2}+\int_{0}^{t} a(s) d s\right),
$$

and ends the proof of Lemma 17

\section{B. Motion failure in the $\pm(1-\cos (x))$ directions for a $2^{\text {nd }}$ order power series expansion}

Throughout all this section, we again assume that 2.5$\rangle$ holds. We now denote by $L^{2}(0, L)$ the space of measurable complex-valued functions such that $\int_{0}^{L}|f|^{2} d x<\infty$. We use the similar convention for $C^{\infty}([0, L]), L^{2}(0, T), H, \mathcal{D}(A)$, mild solutions etc. We also still denote by $\mathcal{P}_{H}$ the orthogonal projection on $H \subset L^{2}(0, L)$ for the hermitian product on $L^{2}(0, L)$. The main result of this section is the following.

Proposition 18. Let $z$ be a mild solution of

$$
\begin{aligned}
& z_{t}+z_{x}+z_{x x x}=0, \\
& z(t, 0)=z(t, L)=0, \\
& z(0, x)=z(T, x)=0,
\end{aligned}
$$

and let $y$ be a mild solution of

$$
\begin{aligned}
& y_{t}+y_{x}+y_{x x x}=0, \\
& y(t, 0)=y(t, L)=0,
\end{aligned}
$$

such that

$$
\int_{0}^{L} y(t, x)(1-\cos (x)) d x=0, \quad \forall t \in[0, T] .
$$


Then

$$
\int_{0}^{T} \int_{0}^{L} y z \sin (x) d x d t=0 .
$$

Before giving the proof of this proposition, let us first mention a corollary:

Corollary 19. Let $T, L, y$ and $z$ be as in Proposition 18 Let $w \in B$ be a mild solution of

$$
\begin{aligned}
& w_{t}+w_{x}+w_{x x x}=-(y z)_{x}, \\
& w(t, 0)=w(t, L)=0,
\end{aligned}
$$

such that

$$
\begin{aligned}
& w(0, x)=0, \\
& \mathcal{P}_{H}(w(T, \cdot))=0 .
\end{aligned}
$$

Then

$$
w(T, x)=0
$$

Indeed, from (B.8) and (B.9), we get

$$
\frac{d}{d t} \int_{0}^{L}(1-\cos (x)) w d x=\int_{0}^{L} y z \sin (x) d x
$$

which, together with $\mathrm{B.7}$ and $\mathrm{B.10}$, gives

$$
\int_{0}^{L}(1-\cos (x)) w(T, x) d x=0 .
$$

Finally, B.12 follows from (B.11) and B.13.

Let us now prove Proposition 18 in two special cases, from which we will then deduce the general case.

Let $\lambda \in \mathbb{C} \backslash \sigma(A)$. Then there exists a unique $y_{\lambda} \in C^{\infty}([0, L])$ such that

$$
\begin{aligned}
& \lambda y_{\lambda}+y_{\lambda x}+y_{\lambda x x x}=0, \\
& y_{\lambda}(0)=y_{\lambda}(L)=0, \\
& y_{\lambda x}(L)=1 .
\end{aligned}
$$

Let

$$
Y_{\lambda}(t, x):=e^{\lambda t} y_{\lambda}(x)
$$

Then the following lemma holds:

Lemma 20. For $\lambda \in \mathbb{C} \backslash \sigma(A)$, the assertion of Theorem 18 holds if

$$
y(t, x):=Y_{\lambda}(t, x) .
$$


(Note that B.14), (B.15), B.17) and (B.18) imply (B.4) and B.5.) Let us prove this lemma. Since $\lambda \in \mathbb{C} \backslash \sigma(A)$, there exists a unique $\phi \in C^{\infty}([0, L])$ such that

$$
\begin{aligned}
& \lambda \phi+\phi_{x}+\phi_{x x x}=y_{\lambda} \sin (x), \\
& \phi(0)=\phi(L)=0, \\
& \phi_{x}(L)=0
\end{aligned}
$$

After some integrations by parts, we get, using (B.1), (B.2), B.19), (B.20), (B.17) and B.18,

$$
\int_{0}^{L} y z \sin (x) d x=\frac{d}{d t} \int_{0}^{L} z \phi e^{\lambda t}-e^{\lambda t}\left[z_{x} \phi_{x}\right]_{0}^{L},
$$

which, together with $\overline{B .3}$ and $(B .21$, gives $B .7]$ if

$$
\phi_{x}(0)=0 .
$$

We multiply (B.19) by $y_{\lambda}(L-x)$ to get, in view of 2.5,

$$
\begin{aligned}
\int_{0}^{L} \lambda \phi y_{\lambda}(L-x)+\int_{0}^{L}\left(\phi_{x}+\phi_{x x x}\right) & y_{\lambda}(L-x) d x \\
& =\int_{0}^{L} y_{\lambda}(L-x) y_{\lambda}(x) \sin (x) d x=0 .
\end{aligned}
$$

Performing integrations by parts in $\mathrm{B} .23$, we get, using $\mathrm{B} .14, \mathrm{~B} .15$, and $\mathrm{B} .20$,

$$
\phi_{x}(0) y_{\lambda x}(L)=0 \text {, }
$$

which, together with $\overline{B .16}$, gives $\overline{B .22}$ and ends the proof of Lemma 20

Let now $\mu \in \sigma(\tilde{A})$ and $\xi \in C^{\infty}([0, L])$ be such that

$$
\begin{aligned}
& \mu \xi+\xi_{x}+\xi_{x x x}=0, \\
& \xi(0)=\xi(L)=0, \\
& \xi_{x}(L)=\xi_{x}(0) .
\end{aligned}
$$

If $\mu=0$, we assume that

$$
\int_{0}^{L}(1-\cos (x)) \xi d x=0 .
$$

Note that $(B .27)$ is implied by $(B .24)$ and $(B .25)$ if $\mu \neq 0$. One has the following lemma.

Lemma 21. The assertion of Proposition 18 holds for

$$
y(t, x):=e^{\mu t} \xi(x)
$$


(Again (B.24) to (B.28) imply (B.4) to B.6).) Let us prove this lemma. Let us first deal with the case where $\mu \neq 0$. Then, since, by [12, Remark 3.6 (ii)],

$$
\sigma(A) \cap \sigma(\tilde{A})=\{0\},
$$

it follows that $\mu \notin \sigma(A)$. Hence

$$
\left(\xi_{x}(L)=0\right) \Rightarrow(\xi=0)
$$

and there exists a unique $\phi \in C^{\infty}([0, L])$ such that

$$
\begin{aligned}
& \mu \phi+\phi_{x}+\phi_{x x x}=\xi \sin (x), \\
& \phi(0)=\phi(L)=0, \\
& \phi_{x}(L)=0 .
\end{aligned}
$$

We then proceed as in the proof of Lemma 20 to get the desired property.

Let us now turn to the case where $\mu=0$. Then B.24 to B.27 imply the existence of $c \in \mathbb{C}$ such that

$$
\xi(x)=c \sin (x)
$$

We now define $\phi \in C^{\infty}([0, L])$ by

$$
\phi(x):=\frac{1}{6}\left(x-x \cos (x)+\frac{1}{2} \sin (2 x)-\sin (x)\right) .
$$

We have

$$
\begin{aligned}
& \phi_{x}+\phi_{x x x}=\sin ^{2}(x)-\frac{1}{3}(1-\cos (x)), \\
& \phi(0)=\phi(L)=\phi_{x}(0)=\phi_{x}(L)=0,
\end{aligned}
$$

which, together with (B.1) and (B.2), leads to

$$
\int_{0}^{L} z \sin ^{2}(x) d x=\frac{1}{3} \int_{0}^{L}(1-\cos (x)) z d x+\frac{d}{d t} \int_{0}^{L} z \phi .
$$

But, from B.1 and B.2, we get

$$
\frac{d}{d t} \int_{0}^{L}(1-\cos (x)) z d x=0
$$

which, together with B.3, gives

$$
\int_{0}^{L}(1-\cos (x)) z d x=0
$$

Equality (B.7) follows from (B.3, B.28, $\mathrm{B} .31, \mathrm{~B} .32$ and $\mathrm{B} .33$. This ends the proof of Lemma 21

Let $\mathcal{E}$ be the subspace of $L^{2}(0, T) \times L^{2}(0, L)$ spanned by the following pairs:

- $\left(Y_{\lambda x}(\cdot, L), Y_{\lambda}(0, \cdot)\right)$ with $\lambda \in \mathbb{C} \backslash \sigma(A)$,

- $\left(e^{\mu t} \xi_{x}(L), \xi\right)$ with $\mu \in \sigma(\tilde{A})$ and $\xi \in C^{\infty}([0, L])$ satisfying (B.24) to B.27). 
Let us point out that, as in Section 2, we still have 2.50). Therefore $\mathcal{E} \subset L^{2}(0, T) \times H$. We have

Lemma 22. For every $T>0, \mathcal{E}$ is dense in $L^{2}(0, T) \times H$.

Let us prove this lemma. Let $v \in L^{2}(0, T)$ and $h \in L^{2}(0, L)$ be such that $(v, h) \in \mathcal{E}^{\perp}$. Thus, for all $\lambda \in \mathbb{C} \backslash \sigma(A)$,

$$
\int_{0}^{T} \bar{v}(t) e^{\lambda t} d t+\int_{0}^{L} \bar{h}(x) y_{\lambda}(x) d x=0 .
$$

Let us give an estimate on $y_{\lambda}$ in order to prove that $v=0$. We multiply (B.14) by $\bar{y}_{\lambda}$, take the real part and integrate on $[0, L]$. Then, using integrations by parts together with (B.15) and B.16, we get

$$
2 \operatorname{Re}(\lambda) \int_{0}^{L}\left|y_{\lambda}\right|^{2} d x=1-\left|y_{\lambda x}(0)\right|^{2} .
$$

From this equality we deduce that

$$
\left\|y_{\lambda}\right\|_{L^{2}(0, L)} \leq \frac{1}{2 \operatorname{Re}(\lambda)} \quad \text { if } \operatorname{Re}(\lambda)>0 .
$$

The same computations show that, if $w \in \mathcal{D}(A)$ satisfies $A w=\lambda w$ with $\lambda \in \mathbb{C}$, then $2 \operatorname{Re}(\lambda) \int_{0}^{L}|w|^{2} d x=-\left|w_{x}(0)\right|^{2}$. Therefore $\sigma(A) \subset\{\lambda \in \mathbb{C} ; \operatorname{Re}(\lambda) \leq 0\}$. Hence by B.34) and (B.36) the holomorphic function $\lambda \in \mathbb{C} \mapsto \int_{0}^{T} \bar{v}(t) e^{\lambda t}$ is bounded and converges to 0 as $\operatorname{Re}(\lambda)$ tends to $+\infty$. Therefore this holomorphic function is identically equal to 0 , which implies that $v=0$.

Let us now prove that $h \in H^{\perp}$. We have

$$
\int_{0}^{T} \bar{h} \xi d x=0
$$

for every $\xi \in C^{\infty}([0, L])$ satisfying (B.24] for some $\mu \in \sigma(\tilde{A})$ and (B.25) to (B.27]. But, since $i \tilde{A}$ is selfadjoint with compact resolvent, it follows from the spectral decomposition of such operators that the vector space spanned by such $\xi$ is dense in $H$. Hence, by (B.37), $h \in H^{\perp}$.

Let us now end the proof of Proposition 18 by a density argument on $y$. Let $z$ be as in the hypotheses of Proposition 18 Let

$$
F: L^{2}(0, T) \times H \rightarrow \mathbb{C}, \quad(u, \phi) \mapsto \int_{0}^{T} \int_{0}^{L} y z d x d t,
$$

where $y$ is the mild solution of

$$
y_{t}+y_{x}+y_{x x x}=0, \quad y(t, 0)=y(t, L)=0, y_{x}(t, L)=u(t), y(0, x)=\phi(x) .
$$

By Lemmas 20 and 21, this linear map $F$ vanishes on $\mathcal{E}$. By Proposition $7, F$ is continuous. Hence $F$ vanishes on the closure of $\mathcal{E}$, which, by Lemma 22 , is equal to $L^{2}(0, T) \times H$. This ends the proof of Proposition 18. 


\section{Proof of Lemma11}

Let $b \in \mathbb{R} \backslash[-1 / \sqrt{3}, 1 / \sqrt{3}]$ be such that $-2 \lambda=2 i b\left(4 b^{2}-1\right)$. We have

$$
y_{-2 \lambda}=\eta e^{\left(-\sqrt{3 b^{2}-1}-i b\right) x}+(1-\eta) e^{\left(\sqrt{3 b^{2}-1}-i b\right) x}-e^{2 i b x}
$$

with

$$
\eta:=\frac{e^{2 i b L}-e^{\left(\sqrt{3 b^{2}-1}-i b\right) L}}{e^{\left(-\sqrt{3 b^{2}-1}-i b\right) L}-e^{\left(\sqrt{3 b^{2}-1}-i b\right) L}} .
$$

After some integrations by parts, we get, using (2.47) for $-2 \lambda$ in place of $\lambda$, 2.65) and (2.73),

$$
\begin{aligned}
\int_{0}^{L}\left(2 \lambda\left(\delta_{\lambda}-\psi_{\lambda}\right)+\left(\delta_{\lambda}-\psi_{\lambda}\right)_{x}\right. & \left.+\left(\delta_{\lambda x x x}-\psi_{\lambda x x x}\right)\right) y_{-2 \lambda} d x \\
& =-\left(\delta_{\lambda x}(L)-\psi_{\lambda x}(L)\right)\left(y_{-2 \lambda x}(L)-y_{-2 \lambda x}(0)\right) .
\end{aligned}
$$

Thus, by (2.64) and 2.72),

$$
\int_{0}^{L}\left(z_{\lambda} \sin (x)-y_{\lambda} \phi_{\lambda x}\right) y_{-2 \lambda} d x=\left(\delta_{\lambda x}(L)-\psi_{\lambda x}(L)\right)\left(y_{-2 \lambda x}(L)-y_{-2 \lambda x}(0)\right) .
$$

Then, in order to prove Lemma 11 . one just just needs to check that

$$
a \mapsto \int_{0}^{L}\left(z_{\lambda} \sin (x)-y_{\lambda} \phi_{\lambda x}\right) y_{-2 \lambda} d x \text { is not identically } 0 \text { on } \Sigma .
$$

Straightforward computations give

$$
\begin{aligned}
\phi_{\lambda}(x)= & -\frac{1}{12 a(2 a+1)(2 a-1)}\left[\kappa \left[\left(a+1-i \sqrt{3 a^{2}-1}\right) e^{\left(-\sqrt{3 a^{2}-1}-i a+i\right) x}\right.\right. \\
& \left.+\left(a-1-i \sqrt{3 a^{2}-1}\right) e^{\left(-\sqrt{3 a^{2}-1}-i a-i\right) x}\right] \\
& +(1-\kappa)\left[\left(a+1+i \sqrt{3 a^{2}-1}\right) e^{\left(\sqrt{3 a^{2}-1}-i a+i\right) x}\right. \\
& \left.+\left(a-1+i \sqrt{3 a^{2}-1}\right) e^{\left(\sqrt{3 a^{2}-1}-i a-i\right) x}\right] \\
& \left.+\left[(2 a-1) e^{(2 i a+i) x}+(2 a+1) e^{(2 i a-i) x}\right]\right] \\
& +K_{1} e^{2 i a x}+K_{2} e^{\left(-\sqrt{3 a^{2}-1}-i a\right) x}+K_{3} e^{\left(\sqrt{3 a^{2}-1}-i a\right) x}
\end{aligned}
$$

with

where

$$
K_{1}:=\frac{1}{3(2 a+1)(2 a-1)}, \quad K_{2}:=\frac{A}{B}, \quad K_{3}:=\frac{C}{D},
$$

$$
\begin{aligned}
A:= & \left(6 i a^{2}-i+2 a \sqrt{3 a^{2}-1}\right) e^{-2 i a L}+\left(-6 i a^{2}+i-2 a \sqrt{3 a^{2}-1}\right) e^{L\left(-\sqrt{3 a^{2}-1}+i a\right)} \\
& +\left(6 i a^{2}-2 i-2 a \sqrt{3 a^{2}-1}\right) e^{4 i a L}+\left(-i-4 a \sqrt{3 a^{2}-1}\right) e^{2 L\left(\sqrt{3 a^{2}-1}-i a\right)} \\
& +\left(3 i-6 i a^{2}+6 a \sqrt{3 a^{2}-1}\right) e^{L\left(\sqrt{3 a^{2}-1}+i a\right)},
\end{aligned}
$$




$$
\begin{aligned}
& B:= 6 a(2 a+1)(2 a-1)\left(e^{L\left(-\sqrt{3 a^{2}-1}-i a\right)}-e^{L\left(\sqrt{3 a^{2}-1}-i a\right)}\right) \\
& \cdot\left(\left(-3 i a+\sqrt{3 a^{2}-1}\right) e^{-L\left(\sqrt{3 a^{2}-1}+i a\right)}\right. \\
&\left.-2 \sqrt{3 a^{2}-1} e^{2 i a L}+\left(3 i a+\sqrt{3 a^{2}-1}\right) e^{L\left(\sqrt{3 a^{2}-1}-i a\right)}\right), \\
& C:=\left(-i+4 a \sqrt{3 a^{2}-1}\right) e^{L\left(-\sqrt{3 a^{2}-1}-i a\right)}+\left(6 i a^{2}-i-2 a \sqrt{3 a^{2}-1}\right) e^{L\left(\sqrt{3 a^{2}-1}-i a\right)} \\
&\left.+\left(-6 i a^{2}+2 i-2 a \sqrt{3 a^{2}-1}\right) e^{2 i a L}\right]\left[e^{L\left(-\sqrt{3 a^{2}-1}-i a\right)}-e^{2 i a L}\right], \\
& D:=6 a(2 a+1)(2 a-1)\left(e^{L\left(-\sqrt{3 a^{2}-1}-i a\right)}-e^{L\left(\sqrt{3 a^{2}-1}-i a\right)}\right) \\
& \cdot\left(\left(-3 i a+\sqrt{3 a^{2}-1}\right) e^{-L\left(\sqrt{3 a^{2}-1}+i a\right)}\right. \\
&\left.-2 \sqrt{3 a^{2}-1} e^{2 i a L}+\left(3 i a+\sqrt{3 a^{2}-1}\right) e^{L\left(\sqrt{3 a^{2}-1}-i a\right)}\right) .
\end{aligned}
$$

We also have

$$
\begin{aligned}
z_{\lambda}(x)= & k_{1}(x)+\left(-k_{1}(0)+\frac{k_{1}(L)-k_{1}(0)}{e^{\left(\sqrt{3 b^{2}-1}+i b\right) L}-e^{\left(-\sqrt{3 b^{2}-1}+i b\right) L}}\right) e^{\left(-\sqrt{3 b^{2}-1}+i b\right) x} \\
& \left.-\frac{k_{1}(L)-k_{1}(0)}{e^{\left(\sqrt{3 b^{2}-1}+i b\right) L}-e^{\left(-\sqrt{3 b^{2}-1}+i b\right) L}}\right) e^{\left(\sqrt{3 b^{2}-1}+i b\right) x} \\
& +M_{\lambda}\left(-e^{2 i b x}+\bar{\eta} e^{\left(-\sqrt{3 b^{2}-1}+i b\right) x}+(1-\bar{\eta}) e^{\left(\sqrt{3 b^{2}-1}+i b\right) x}\right),
\end{aligned}
$$

where $M_{\lambda} \in \mathbb{C}$ is a constant and

$$
\begin{aligned}
k_{1}(x):= & -\frac{\kappa^{2} i\left(-\sqrt{3 a^{2}-1}-i a\right)}{2\left(24 a^{3}-9 a+3 i \sqrt{3 a^{2}-1}\right)} e^{\left(-2 \sqrt{3 a^{2}-1}-2 i a\right) x} \\
& -\frac{(1-\kappa)^{2} i\left(\sqrt{3 a^{2}-1}-i a\right)}{2\left(24 a^{3}-9 a-3 i \sqrt{3 a^{2}-1}\right)} e^{\left(2 \sqrt{3 a^{2}-1}-2 i a\right) x} \\
& +\frac{\kappa(1-\kappa)}{12 a^{2}-3} e^{-2 i a x}+\frac{e^{4 i a x}}{24 a^{2}}-\frac{\kappa i\left(-\sqrt{3 a^{2}-1}+i a\right)}{2\left(12 a^{3}-3 a\right)} e^{\left(-\sqrt{3 a^{2}-1}+i a\right) x} \\
& -\frac{i(1-\kappa)\left(\sqrt{3 a^{2}-1}+i a\right)}{2\left(12 a^{3}-3 a\right)} e^{\left(\sqrt{3 a^{2}-1}+i a\right) x} .
\end{aligned}
$$

Since

$$
\begin{array}{r}
\int_{0}^{L}\left(-e^{2 i b x}+\bar{\eta} e^{\left(-\sqrt{3 b^{2}-1}+i b\right) x}+(1-\bar{\eta}) e^{\left(\sqrt{3 b^{2}-1}+i b\right) x}\right) y_{-2 \lambda} \sin (x) d x \\
=\int_{0}^{L} \bar{y}_{-2 \lambda} y_{-2 \lambda} \sin (x) d x=0,
\end{array}
$$

one does not need to know $M_{\lambda}$ to compute the integral in C.11. Let

$$
Q:=E \int_{0}^{L}\left(z_{\lambda} \sin (x)-y_{\lambda} \phi_{\lambda x}\right) y_{-2 \lambda} d x,
$$


with

$$
\begin{aligned}
E= & \left(e^{L\left(-\sqrt{3 a^{2}-1}-i a\right)}-e^{L\left(\sqrt{3 a^{2}-1}-i a\right)}\right)\left(e^{L\left(\sqrt{3 b^{2}-1}+i b\right)}-e^{L\left(-\sqrt{3 b^{2}-1}+i b\right)}\right) \\
& \cdot\left(\left(-3 i a+\sqrt{3 a^{2}-1}\right) e^{-L\left(\sqrt{3 a^{2}-1}+i a\right)}-2 \sqrt{3 a^{2}-1} e^{2 i a L}\right. \\
& \left.+\left(3 i a+\sqrt{3 a^{2}-1}\right) e^{L\left(\sqrt{3 a^{2}-1}+i a\right)}\right) .
\end{aligned}
$$

Then $Q$ is a finite sum of terms of the form $P_{c}(a) e^{\left(c_{1} \sqrt{3 a^{2}-1}+c_{2} i a+c_{3} \sqrt{3 b^{2}-1}+c_{4} i b\right) L}$ where $P_{c}(a)$ is a rational function of $a, b, \sqrt{3 a^{2}-1}$ and $\sqrt{3 b^{2}-1}$ and $c:=\left(c_{1}, c_{2}, c_{3}, c_{4}\right) \in$ $\mathbb{Z}^{4}$. (There are 110 such terms.) It is easy to prove that C.1 is identically 0 (if and) only if every $P_{c}$ is identically 0 .

We look at $P_{(3,1,2,2)}$. After lengthy but straightforward computations, we get

$$
\begin{aligned}
P_{(3,1,2,2)}(a)= & -\left(3 i a+\sqrt{3 a^{2}-1}\right)\left(f_{1}+f_{2}+f_{3}+f_{4}+f_{5}+f_{6}+f_{7}+f_{8}+f_{9}\right) \\
& +\frac{6 i a^{2}-i-2 a \sqrt{3 a^{2}-1}}{6 a(2 a+1)(2 a-1)}\left(f_{10}+f_{11}\right),
\end{aligned}
$$

with

$$
\begin{aligned}
& f_{1}=\frac{\left(\sqrt{3 a^{2}-1}-i a\right) i}{48 a^{3}-18 a-6 i \sqrt{3 a^{2}-1}}\left(\frac{1}{\left(2 \sqrt{3 a^{2}-1}-2 i a+\sqrt{3 b^{2}-1}-i b\right)^{2}+1}\right. \\
& \left.-\frac{1}{\left(2 \sqrt{3 a^{2}-1}-2 i a+2 i b\right)^{2}+1}\right) \text {, } \\
& f_{2}=\frac{-1}{24 a^{2}}\left(\frac{1}{\left(4 i a+\sqrt{3 b^{2}-1}-i b\right)^{2}+1}-\frac{1}{(4 i a+2 i b)^{2}+1}\right) \text {, } \\
& f_{3}=\frac{\left(\sqrt{3 a^{2}-1}+i a\right) i}{24 a^{3}-6 a}\left(\frac{1}{\left(\sqrt{3 a^{2}-1}+i a+\sqrt{3 b^{2}-1}-i b\right)^{2}+1}\right. \\
& \left.-\frac{1}{\left(\sqrt{3 a^{2}-1}+i a+2 i b\right)^{2}+1}\right) \\
& f_{4}=\frac{1}{12 a(2 a+1)(2 a-1)} \\
& \cdot\left[\left(\frac{a+1+i \sqrt{3 a^{2}-1}}{2 \sqrt{3 a^{2}-1}-2 i a+i+\sqrt{3 b^{2}-1}-i b}\right.\right. \\
& \left.+\frac{a-1+i \sqrt{3 a^{2}-1}}{2 \sqrt{3 a^{2}-1}-2 i a-i+\sqrt{3 b^{2}-1}-i b}\right) \\
& \left.-\left(\frac{a+1+i \sqrt{3 a^{2}-1}}{2 \sqrt{3 a^{2}-1}-2 i a+i+2 i b}+\frac{a-1+i \sqrt{3 a^{2}-1}}{2 \sqrt{3 a^{2}-1}-2 i a-i+2 i b}\right)\right] \text {, }
\end{aligned}
$$




$$
\begin{aligned}
& f_{5}=-\frac{1}{12 a(2 a+1)(2 a-1)} \\
& \cdot\left[\left(\frac{a+1+i \sqrt{3 a^{2}-1}}{\sqrt{3 a^{2}-1}+i a+i+\sqrt{3 b^{2}-1}-i b}\right.\right. \\
&\left.+\frac{a-1+i \sqrt{3 a^{2}-1}}{\sqrt{3 a^{2}-1}+i a-i+\sqrt{3 b^{2}-1}-i b}\right) \\
&\left.-\left(\frac{a+1+i \sqrt{3 a^{2}-1}}{\sqrt{3 a^{2}-1}+i a+i+2 i b}+\frac{a-1+i \sqrt{3 a^{2}-1}}{\sqrt{3 a^{2}-1}+i a-i+2 i b}\right)\right] \\
& f_{6}= \frac{i}{12 a}\left[\left(\frac{1}{\sqrt{3 a^{2}-1}+i a+i+\sqrt{3 b^{2}-1}-i b}\right.\right. \\
&\left.+\frac{1}{\sqrt{3 a^{2}-1}+i a-i+\sqrt{3 b^{2}-1}-i b}\right) \\
&\left.-\left(\frac{1}{\sqrt{3 a^{2}-1}+i a+i+2 i b}+\frac{1}{\sqrt{3 a^{2}-1}+i a-i+2 i b}\right)\right] \\
& f_{7}= \frac{-i}{12 a}\left[\left(\frac{1}{4 i a+i+\sqrt{3 b^{2}-1}-i b}+\frac{1}{4 i a-i+\sqrt{3 b^{2}-1}-i b}\right)\right. \\
&\left.-\left(\frac{1}{4 i a+i+2 i b}+\frac{1}{4 i a-i+2 i b}\right)\right] \\
& f_{8}= \frac{-2 i a}{3(2 a+1)(2 a-1)} \\
& \cdot\left[\frac{1}{\sqrt{3 a^{2}-1}+i a+\sqrt{3 b^{2}-1}-i b}-\frac{1}{\sqrt{3 a^{2}-1}+i a+2 i b}\right] \\
& f_{11}=\left(\sqrt{3 a^{2}-1}-i a\right)\left[\frac{1}{\sqrt{3 a^{2}-1}+i a+\sqrt{3 b^{2}-1}-i b}-\frac{1}{\sqrt{3 a^{2}-1}+i a+2 i b}\right] \\
& f_{10}=-\left(\sqrt{3 a^{2}-1}-i a\right) \\
& \cdot\left[\frac{1}{2 \sqrt{3 a^{2}-1}-2 i a+\sqrt{3 b^{2}-1}-i b}-\frac{1}{4 i a+\sqrt{3 a^{2}-1}-2 i b}\right] \\
& \hline
\end{aligned}
$$

As $a \rightarrow+\infty$, one concludes that $P_{(3,1,2,2)}(a) \sim p / a$ with

$$
\begin{aligned}
p:=\frac{(\sqrt{3}-i)(6 i-2 \sqrt{3})}{24} & \left(-\frac{1}{2 \sqrt{3}-2 i+2^{1 / 3} \sqrt{3}+i 2^{1 / 3}}+\frac{1}{2 \sqrt{3}-2 i-i 2^{4 / 3}}\right. \\
+ & \left.\frac{1}{\sqrt{3}+i+2^{1 / 3} \sqrt{3}+i 2^{1 / 3}}-\frac{1}{\sqrt{3}+i-i 2^{4 / 3}}\right) \neq 0 .
\end{aligned}
$$

In particular $P_{(3,1,2,2)}$ is not identically equal to 0 . 


\section{References}

[1] Agrachev, A.: Newton diagrams and tangent cones to attainable sets. In: Analysis of Controlled Dynamical Systems (Lyon 1990), B. Bonnard et al. (eds.), Progr. Systems Control Theory 8, Birkhäuser, Boston, 11-20 (1991) Zbl 0811.93006 MR 92h:93012

[2] Bianchini, R. M., Stefani, G.: Controllability along a trajectory: a variational approach. SIAM J. Control Optim. 31, 900-927 (1993) Zbl 0797.49015 MR 94d:93009

[3] Bona, J., Winther, R.: The Korteweg-de Vries equation posed in a quarter-plane. SIAM J. Math. Anal. 14, 1056-1106 (1983) Zbl 0529.35069 MR 85c:35076

[4] Coron, J.-M.: Contrôlabilité exacte frontière de l'équation d'Euler des fluides parfaits incompressibles bidimensionnels. C. R. Acad. Sci. Paris 317, 271-276 (1993) Zbl 0781.76013 MR 94g:93067

[5] Coron, J.-M.: On the controllability of 2-D incompressible perfect fluids. J. Math. Pures Appl. 75, 155-188 (1996) Zbl 0848.76013 MR 97b:93010

[6] Coron, J.-M.: Local controllability of a 1-D tank containing a fluid modeled by the shallow water equations. ESAIM Control Optim. Calc. Var. 8, 513-554 (2002) MR 2004a:93009

[7] Coron, J.-M., Trélat, E.: Global steady-state controllability of 1-D semilinear heat equations. Preprint, Université Paris-Sud, 2003, submitted to SIAM J. Control Optim.

[8] Crépeau, E.: Exact controllability of the Korteweg-de Vries equation around a non-trivial stationary solution. Internat. J. Control 74, 1096-1106 (2001) Zbl 1016.93031

[9] Hermes, H.: Controlled stability. Ann. Mat. Pura Appl. 114, 103-119(1977) Zbl 0385.9301 MR 58 \#3067

[10] Kawski, M.: High-order small time local controllability. In: Nonlinear Controllability and Optimal Control, H. J. Sussmann (ed.), Monogr. Textbooks Pure Appl. Math. 133, Dekker, New York, 431-467 (1990). Zbl 0703.93013 MR 91j:93010

[11] Micu, S., Ortega, J. H.: On the controllability of a linear coupled system of Korteweg-de Vries equations. In: Mathematical and Numerical Aspects of Wave Propagation (Santiago de Compostela, 2000), SIAM, Philadelphia PA, 1020-1024 (2000). Zbl 0958.93046 MR 1786022

[12] Rosier, L.: Exact boundary controllability for the Korteweg-de Vries equation on a bounded domain. ESAIM Control Optim. Calc. Var. 2, 33-55 (1997) Zbl 0873.93008 MR 98d:93016

[13] Rosier, L.: Exact boundary controllability for the linear Korteweg-de Vries equations on a bounded domain. ESAIM Proc. 4, 255-267 (1998) Zbl 0919.93039 MR 99i:93008

[14] Rosier, L.: Exact boundary controllability for the linear Korteweg-de Vries equation on the half-line. SIAM J. Control Optim. 39, 331-351 (2000) Zbl 0966.93055 MR 2001j:9301

[15] Rosier, L.: Control of the surface of a fluid by a wavemaker. ESAIM Control Optim. Calc. Var., submitted

[16] Russell, D. L., Yang, B.-Y.: Exact controllability and stabilizability of the Kortewegde Vries equation. Trans. Amer. Math. Soc. 348, 3643-3672 (1996) Zbl 0862.93035 MR 96m:93025

[17] Sussmann, H. J.: Lie brackets and local controllability: a sufficient condition for scalar-input systems. SIAM J. Control Optim. 21, 686-713 (1983) Zbl 0523.49026 MR 85j:49029

[18] Sussmann, H. J.: A general theorem on local controllability. SIAM J. Control Optim. 25, 158-194 (1987) Zbl 0629.93012 MR 88f:93025

[19] Whitham, G. B.: Linear and Nonlinear Waves. Wiley, New York, 1974. Zbl 0373.76001 MR 2000c:35001

[20] Tret'yak, A. I.: On odd-order necessary conditions for optimality in a time-optimal control problem for systems linear in the control. Math. USSR-Sb. 70, 47-63 (1991) Zbl 0731.49022 